\title{
INVARIANTS OF LIMIT KEY POLYNOMIALS
}

\author{
MARIA ALBERICH-CARRAMIÑANA, ALBERTO F. BOIX, JULIO FERNÁNDEZ, JORDI GUÀRDIA, \\ ENRIC NART, AND JOAQUIM ROÉ
}

\begin{abstract}
Let $\nu$ be a valuation of arbitrary rank on the polynomial ring $K[x]$ with coefficients in a field $K$. We prove comparison theorems between MacLane-Vaquié key polynomials for valuations $\mu \leq \nu$ and abstract key polynomials for $\nu$.

Also, some results on invariants associated to limit key polynomials are obtained. In particular, if $\operatorname{char}(K)=0$ we show that all the limit key polynomials of unbounded continuous families of augmentations have the numerical character equal to one.
\end{abstract}

\section{INTRODUCTION}

Consider a valuation $\nu$ on the polynomial ring $K[x]$, with coefficients in a field $K$. Let $\Gamma_{\nu}$ be its value group. The graded algebra of $\nu$ is the integral domain

$$
\operatorname{gr}_{\nu}(K[x])=\bigoplus_{\gamma \in \Gamma_{\nu}} \mathcal{P}_{\gamma} / \mathcal{P}_{\gamma}^{+}
$$

where $\mathcal{P}_{\gamma}=\{g \in K[x] \mid \nu(g) \geq \gamma\} \supset \mathcal{P}_{\gamma}^{+}=\{g \in K[x] \mid \nu(g)>\gamma\}$.

A MacLane-Vaquié (MLV) key polynomial for $\nu$ is a monic polynomial $\phi \in K[x]$ whose initial term generates a prime ideal in the graded algebra $\operatorname{gr}_{\nu}(K[x])$, which cannot be generated by the initial term of a polynomial of smaller degree.

Let $\operatorname{KP}(\nu)$ be the set of MLV key polynomials for $\nu$. If $\operatorname{KP}(\nu) \neq \emptyset$, the minimal degree of a polynomial in $\operatorname{KP}(\nu)$ is called the degree of $\nu$.

By the work of MacLane and Vaquié, we may associate to $\nu$ a countable sequence of augmentations of valuations on $K[x]$

$$
\mu_{0}<\mu_{1}<\cdots<\mu_{n}<\cdots<\nu
$$

containing certain numerical invariants of $\nu[4,14,7]$.

This chain of augmentations of valuations is a mixture of ordinary and limit augmentations. In both cases, certain MLV key polynomials of the intermediate valuations $\mu_{n}<\nu$ are involved.

The initial valuation $\mu_{0}$ has always degree one. Let $\mathcal{K} \mathcal{P}_{0}=\left\{\phi_{0}\right\}$, where $\phi_{0} \in \operatorname{KP}\left(\mu_{0}\right)$ is any MLV key polynomial of degree one.

If $\mu_{n-1}<\mu_{n}$ is an ordinary augmentation, there exists $\phi_{n} \in \operatorname{KP}\left(\mu_{n-1}\right)$ such that $\mu_{n}$ is equal to the truncated valuation $\nu_{\phi_{n}}$. That is, in terms of $\phi_{n}$-expansions of polynomials $f \in K[x]$, the valuation $\mu_{n}$ acts as follows

$$
f=\sum_{0 \leq s} a_{s} \phi_{n}^{s}, \quad \operatorname{deg}\left(a_{s}\right)<\operatorname{deg}\left(\phi_{n}\right) \Longrightarrow \mu_{n}(f)=\min \left\{\nu\left(a_{s} \phi_{n}^{s}\right) \mid 0 \leq s\right\} .
$$

To any such ordinary augmentation step we attach the set

$$
\mathcal{K} \mathcal{P}_{n}=\left\{\phi_{n}\right\} .
$$

2010 Mathematics Subject Classification. Primary 13A18; Secondary 12J20, 13J10, 14E15.

Key words and phrases. continuous family of augmentations, key polynomial, valuation.

Partially supported by grants MTM2016-75980-P, MTM2015-69135-P and PID2019-103849GB-I00 from the Spanish Research Agency, and grant 2017SGR-932 from Generalitat de Catalunya. 
If $\mu_{n-1}<\mu_{n}$ is a limit augmentation, there exists an ordinal $\lambda_{n}$ and a well-ordered set $\left(\chi_{\alpha}\right)_{\alpha<\lambda_{n}}$ of MLV key polynomials for $\mu_{n-1}$ of constant degree $m$, such that the corresponding ordinary augmentations $\rho_{\alpha}=\nu_{\chi_{\alpha}}$ of $\mu_{n-1}$ satisfy

$$
\alpha<\beta \Longrightarrow \rho_{\alpha}<\rho_{\beta}<\mu_{n} \text {. }
$$

A polynomial $f \in K[x]$ is stable with respect to the set $\left(\rho_{\alpha}\right)_{\alpha<\lambda_{n}}$ if there exists an index $\alpha_{0}$ such that $\rho_{\alpha}(f)=\rho_{\alpha_{0}}(f)$ for all $\alpha \geq \alpha_{0}$. In this case, we denote this stable value by $\rho_{\infty}(f)$. Otherwise $f$ is said to be unstable.

In a limit augmentation, all polynomials of degree $m$ are stable, but there are necessarily unstable polynomials. Any unstable monic polynomial $\phi$ of minimal degree is said to be a $M L V$ limit key polynomial for the set $\left(\rho_{\alpha}\right)_{\alpha<\lambda_{n}}$.

The limit augmented valuation $\mu_{n}$ is equal to $\nu_{\phi_{n}}$ for some MLV limit key polynomial.

To any such limit augmentation step, we associate the well-ordered set

$$
\mathcal{K} \mathcal{P}_{n}=\left\{\chi_{\alpha} \mid \alpha<\lambda_{n}\right\}+\left\{\phi_{n}\right\},
$$

where "+" denotes the usual sum of totally ordered sets.

A celebrated result of MacLane-Vaquié states that $\nu$ falls in one, and only one, of the following cases [7, Thm. 4.3].

(a) After a finite number $r$ of augmentation steps, we get $\mu_{r}=\nu$.

(b) After a finite number $r$ of augmentation steps, $\nu$ is the stable limit $\nu=\rho_{\infty}$, of some well-ordered set $\left(\rho_{\alpha}\right)_{\alpha<\lambda}$ of ordinary augmentations of $\mu_{r}$ of constant degree, such that all polynomials in $K[x]$ are stable.

(c) It is the stable limit, $\nu=\lim _{n \rightarrow \infty} \mu_{n}$, of a countably infinite chain of augmentations as in (1), with unbounded degree.

We say that $\nu$ has finite depth $r$, quasi-finite depth $r$, or infinite depth, respectively.

If $\nu$ has quasi-finite depth, consider the totally ordered set

$$
\mathcal{K} \mathcal{P}_{\infty}=\left\{\chi_{\alpha} \mid \alpha<\lambda\right\},
$$

where $\chi_{\alpha} \in \mathrm{KP}\left(\mu_{r}\right)$ is a MLV key polynomial such that $\rho_{\alpha}=\nu_{\chi_{\alpha}}$, for all $\alpha<\lambda$.

Then, the well-ordered set of polynomials:

$$
\mathcal{K P}= \begin{cases}\mathcal{K} \mathcal{P}_{0}+\cdots+\mathcal{K} \mathcal{P}_{r}, & \text { if } \nu \text { has finite depth } r, \\ \mathcal{K} \mathcal{P}_{0}+\cdots+\mathcal{K} \mathcal{P}_{r}+\mathcal{K} \mathcal{P} \infty, & \text { if } \nu \text { has quasi-finite depth } r \\ \mathcal{K} \mathcal{P}_{0}+\cdots+\mathcal{K} \mathcal{P}_{n}+\cdots, & \text { if } \nu \text { has infinite depth, }\end{cases}
$$

is a complete set of key polynomials for $\nu$, as defined by F.J. Herrera Govantes, W. Mahboub, M.A. Olalla Acosta and M. Spivakovsky in [2]. That is, for any $f \in K[x]$ there exists $Q \in \mathcal{K} \mathcal{P}$ such that $\nu(f)=\nu_{Q}(f)$. As a consequence, for any $\gamma \in \Gamma_{\nu}$, the set of polynomials

$$
\mathcal{K} \mathcal{P}_{\gamma}=\left\{a Q_{1}^{n_{1}} \cdots Q_{\ell}^{n_{\ell}} \mid a \in K^{*}, Q_{1}, \ldots, Q_{\ell} \in \mathcal{K} \mathcal{P}, n_{1}, \ldots, n_{\ell} \in \mathbb{N}\right\} \cap \mathcal{P}_{\gamma}
$$

is a set of generators of $\mathcal{P}_{\gamma}$ as an additive group.

This property is the motivation for Spivakovsky's strategy to attack the problem of local uniformization $[8,12]$.

Certain abstract key polynomials were introduced by J. Decaup, W. Mahboub and M. Spivakovsky as an intrinsic characterization of the polynomials in $\mathcal{K} \mathcal{P}$ [1]. This idea was developed by Novacoski and Spivakovsky in $[9,10]$, where they proved some further properties of key polynomials.

In this paper, we have a double aim. On one hand, in section 2, we review some of these results, aiming at a determination of which MLV key polynomials of the intermediate 
valuations $\mu_{n}$ are abstract key polynomials for $\nu$. We complete in this way some partial results from [1, Sec. 3].

In section 3 we obtain similar results for limit key polynomials. Abstract limit key polynomials were introduced by Novacoski and Spivakovsky in [9]. We prove that they coincide with the MLV limit key polynomials of the limit augmentation steps in (1).

On the other hand, in section 4, we obtain some results on invariants attached to limit key polynomials $\phi$ of a continuous family of augmentations

$$
\left(\rho_{\alpha}\right)_{\alpha<\lambda}, \quad \rho_{\alpha}=\left[\mu ; \chi_{\alpha}, \gamma_{\alpha}\right],
$$

of some base valuation $\mu$. Our main result in this section is Theorem 4.11, where we prove an identity between some of these invariants:

$$
t_{\infty}(\phi) b_{\infty}=\operatorname{mult}(\phi)
$$

whenever the set of values $\gamma_{\alpha}=\rho_{\alpha}\left(\chi_{\alpha}\right)$ is unbounded in a suitable group.

The number $\operatorname{mult}(\phi)$ is the least positive integer $b$ such that $\partial_{b}(\phi) \neq 0$, where $\partial_{b}=\frac{1}{b !} \frac{\partial^{b}}{\partial x^{b}}$ is the $b$-th formal derivative, which makes sense in any characteristic.

For any $\alpha$, consider the $\chi_{\alpha}$-expansion $\phi=\sum_{0 \leq s} a_{s, \alpha} \chi_{\alpha}^{s}$ and let $t_{\alpha}(\phi)$ be the maximal index $s$ such that $\rho_{\alpha}(\phi)=\rho_{\alpha}\left(a_{s, \alpha} \chi_{\alpha}^{s}\right)$. This positive integer $t_{\alpha}(\phi)$ stabilizes for $\alpha$ sufficiently large $\left[13\right.$, Sec. 3], [2, Sec. 4]. We denote by $t_{\infty}(\phi)$ the stable value of $t_{\alpha}(\phi)$, which is known as the numerical character of $\phi$.

Finally, for any $\alpha$, let $b_{\alpha}$ be the largest positive integer such that $\left(\nu\left(\chi_{\alpha}\right)-\nu\left(\partial_{b_{\alpha}}\left(\chi_{\alpha}\right)\right) / b_{\alpha}\right.$ takes a maximal value in $\Gamma_{\nu} \otimes \mathbb{Q}$. It is shown in $\left[2\right.$, Sec. 7] that $b_{\alpha}$ stabilizes for $\alpha$ sufficiently large. We define $b_{\infty}$ to be the stable value of $b_{\alpha}$.

As a consequence of (2), if $\operatorname{char}(K)=0$, then $t_{\infty}(\phi)=b_{\infty}=1$, because mult $(\phi)=1$.

\section{Preliminaries}

1.1. Valuations on a polynomial ring. Consider a valued field $(K, v)$. Let $k$ be the class field of the valuation ring and $\Gamma=v\left(K^{*}\right)$ the value group. Denote the divisible hull of $\Gamma$ by

$$
\Gamma_{\mathbb{Q}}=\Gamma \otimes \mathbb{Q} \text {. }
$$

Let $K[x]$ be the polynomial ring in one indeterminate. All the valuations on $K[x]$ considered in this paper are assumed to extend this fixed valuation $v$ on $K$.

Consider a valuation $\nu$ on $K[x]$. That is, for some embedding $\Gamma \hookrightarrow \Lambda$ into another ordered abelian group, we consider a mapping

$$
\nu: K[x] \longrightarrow \Lambda \cup\{\infty\}
$$

whose restriction to $K$ is $v$, and which satisfies the following two conditions:

(1) $\nu(f g)=\nu(f)+\nu(g), \quad \forall f, g \in K[x]$.

(2) $\nu(f+g) \geq \min \{\nu(f), \nu(g)\}, \quad \forall f, g \in K[x]$.

The support of $\nu$ is the prime ideal

$$
\mathfrak{p}=\nu^{-1}(\infty) \in \operatorname{Spec}(K[x])
$$

The value group of $\nu$ is the subgroup $\Gamma_{\nu} \subset \Lambda$ generated by $\nu(K[x] \backslash \mathfrak{p})$.

The valuation $\nu$ induces a valuation on the residue field $\kappa(\mathfrak{p})$, the field of fractions of $K[x] / \mathfrak{p}$. Let $k_{\nu}$ be the residue class field of this valuation on $\kappa(\mathfrak{p})$.

Clearly, $\kappa(0)=K(x)$, while for $\mathfrak{p} \neq 0$ the field $\kappa(\mathfrak{p})$ is a simple finite extension of $K$.

The extension $\nu / v$ is commensurable if $\Gamma_{\nu} / \Gamma$ is a torsion group. In this case, there is a canonical embedding $\Gamma_{\nu} \hookrightarrow \Gamma_{\mathbb{Q}}$. All valuations with non-trivial support are commensurable over $v$. 
We denote the graded algebra of $\nu$ defined in the Introduction by

$$
\mathcal{G}_{\nu}=\operatorname{gr}_{\nu}(K[x]) \text {. }
$$

If $\nu$ has non-trivial support $\mathfrak{p} \neq 0$, there is a natural isomorphism of graded algebras

$$
\mathcal{G}_{\nu} \simeq \operatorname{gr}_{\bar{\nu}}(\kappa(\mathfrak{p})),
$$

where $\bar{\nu}$ is the valuation on $\kappa(\mathfrak{p})$ induced by $\nu$.

Consider the initial term mapping $\operatorname{in}_{\nu}: K[x] \rightarrow \mathcal{G}_{\nu}$, given by $\operatorname{in}_{\nu} 0=0$ and

$$
\operatorname{in}_{\nu} g=g+\mathcal{P}_{\nu(g)}^{+} \in \mathcal{P}_{\nu(g)} / \mathcal{P}_{\nu(g)}^{+},
$$

if $g \neq 0$. The following definitions translate properties of the action of $\nu$ on $K[x]$ into algebraic relationships in the graded algebra $\mathcal{G}_{\nu}$.

Definition 1.1. Let $g, h \in K[x]$.

We say that $g, h$ are $\nu$-equivalent, and we write $g \sim_{\nu} h$, if $\operatorname{in}_{\nu} g=\operatorname{in}_{\nu} h$.

We say that $g$ is $\nu$-divisible by $h$, and we write $\left.h\right|_{\nu} g$, if $\operatorname{in}_{\nu} h \mid \operatorname{in}_{\nu} g$ in $\mathcal{G}_{\nu}$.

1.2. MacLane-Vaquié key polynomials. A polynomial $g \in K[x]$ is $\nu$-irreducible if $\left(\operatorname{in}_{\nu} g\right) \mathcal{G}_{\nu}$ is a non-zero prime ideal.

We say that $g$ is $\nu$-minimal if $g \nmid \nu f$ for all non-zero $f \in K[x]$ with $\operatorname{deg}(f)<\operatorname{deg}(g)$.

For an arbitrary $g \in K[x]$ we may define the truncation $\nu_{g}$ as we did in the introduction for key polynomials:

$$
f=\sum_{0 \leq s} a_{s} g^{s}, \quad \operatorname{deg}\left(a_{s}\right)<\operatorname{deg}(g) \Longrightarrow \nu_{g}(f)=\min \left\{\nu\left(a_{s} g^{s}\right) \mid 0 \leq s\right\} .
$$

This function $\nu_{g}$ is not necessarily a valuation, but it is useful to characterize the $\nu$ minimality of $g$.

Lemma 1.2. [6, Prop. 2.3] A polynomial $g \in K[x] \backslash K$ is $\nu$-minimal if and only if $\nu_{g}=\nu$.

A MacLane-Vaquié (MLV) key polynomial for $\nu$ is a monic polynomial in $K[x]$ which is $\nu$-minimal and $\nu$-irreducible. A MLV key polynomial is necessarily irreducible in $K[x]$.

We recall that $\operatorname{KP}(\nu)$ denotes the set of MLV key polynomials for $\nu$.

Suppose that $\nu$ has non-trivial support. By the isomorphism of (3), every non-zero homogeneous element of $\mathcal{G}_{\nu}$ is a unit. Therefore, no polynomial in $K[x]$ can be $\nu$-irreducible. Thus, $\operatorname{KP}(\nu)=\emptyset$.

If $\operatorname{KP}(\nu) \neq \emptyset$, the following subset of $\Gamma_{\nu}$ is a subgroup:

$$
\Gamma_{\nu, \operatorname{deg}(\nu)}=\{\nu(a) \mid 0 \leq \operatorname{deg}(a)<\operatorname{deg}(\nu)\},
$$

where $\operatorname{deg}(\nu)$ is the minimal degree of the polynomials in $\operatorname{KP}(\nu)$ [6, Lem. 2.11].

Definition 1.3. The relative ramification index of $\nu$ is the index $e_{\text {rel }}(\nu)=\left(\Gamma_{\nu}: \Gamma_{\nu, \operatorname{deg}(\nu)}\right)$.

Consider the subring of homogeneous elements of degree zero in the graded algebra

$$
\Delta=\Delta_{\nu}=\mathcal{P}_{0} / \mathcal{P}_{0}^{+} \subset \mathcal{G}_{\nu}
$$

There are canonical injective ring homomorphisms $k \hookrightarrow \Delta \hookrightarrow k_{\nu}$. We denote the algebraic closure of $k$ in $\Delta$ by

$$
\kappa=\kappa(\nu) \subset \Delta .
$$

This is a subfield such that $\kappa^{*}=\Delta^{*}$, the multiplicative group of all units in $\Delta$.

Theorem 1.4. [6, Thm. 4.4] The set $\mathrm{KP}(\nu)$ is empty if and only if all the homogeneus elements in $\mathcal{G}_{\nu}$ are units. Equivalently, $\nu / v$ is commensurable and $\kappa=\Delta=k_{\nu}$ is an algebraic extension of $k$. 
For any $\phi \in \mathrm{KP}(\nu)$, we denote by $[\phi]_{\nu} \subset \mathrm{KP}(\nu)$ the subset of all MLV key polynomials which are $\nu$-equivalent to $\phi$.

Theorem 1.5. [6, Thm. 4.2] Suppose $\nu / v$ incommensurable. Let $\phi \in K[x]$ be a monic polynomial of minimal degree satisfying $\nu(\phi) \notin \Gamma_{\mathbb{Q}}$. Then, $\phi$ is a $M L V$ key polynomial for $\nu$, and $\operatorname{KP}(\nu)=[\phi]_{\nu}$. In this case, $\kappa=\Delta=k_{\nu}$ is a finite extension of $k$.

Theorem 1.6. [6, Thms. 4.5,4.6] Suppose $\nu / v$ commensurable and $\operatorname{KP}(\nu) \neq \emptyset$. Let $\phi$ be a $M L V$ key polynomial for $\nu$ of minimal degree $m$. Let $e=e_{\text {rel }}(\nu)$.

Let $u=\operatorname{in}_{\nu} a \in \mathcal{G}_{\nu}^{*}$, for some $a \in K[x]$ such that $\operatorname{deg}(a)<m$ and $\nu(a)=e \nu(\phi)$. Then, $\xi=\left(\mathrm{in}_{\nu} \phi\right)^{e} u^{-1} \in \Delta$ is transcendental over $k$ and satisfies $\Delta=\kappa[\xi]$.

Moreover, the canonical embedding $\Delta \hookrightarrow k_{\nu}$ induces an isomorphism $\kappa(\xi) \simeq k_{\nu}$.

These comensurable extensions $\nu / v$ admitting MLV key polynomials are called residually transcendental valuations on $K[x]$.

The pair $\phi, u$ determines a (non-canonical) residual polynomial operator

$$
R=R_{\nu, \phi, u}: K[x] \longrightarrow \kappa[y],
$$

whose images are monic polynomials in the indeterminate $y$, which are not divisible by $y$ [6, Sec. 5]. This operator facilitates a complete description of the set $\operatorname{KP}(\nu)$.

Theorem 1.7. [6, Prop. 6.3] Suppose $\nu / v$ commensurable and $\operatorname{KP}(\nu) \neq \emptyset$. Let $\phi$ be a $M L V$ key polynomial for $\nu$ of minimal degree $m$. A monic $\chi \in K[x]$ is a key polynomial for $\nu$ if and only if either

(1) $\operatorname{deg}(\chi)=m$ and $\chi \sim_{\nu} \phi$, or

(2) $\operatorname{deg}(\chi)=$ medeg $(R(\chi))$ and $R(\chi)$ is irreducible in $\kappa[y]$.

Moreover, $\chi, \chi^{\prime} \in \mathrm{KP}(\nu)$ are $\nu$-equivalent if and only if $R(\chi)=R\left(\chi^{\prime}\right)$. In this case, $\operatorname{deg}(\chi)=\operatorname{deg}\left(\chi^{\prime}\right)$.

The set $\operatorname{KP}(\nu) / \sim_{\nu}$ is in canonical bijection with the maximal spectrum of $\Delta[6$, Thm. 6.7]. Since the choice of a pair $\phi, u$ as above determines an isomorphism $\Delta \simeq \kappa[y]$, it induces a (non-canonical) bijection between $\mathrm{KP}(\nu) / \sim_{\nu}$ and the set of monic irreducible polynomials in $\kappa[y]$.

1.3. Chains of valuations. Let us fix an embedding $\Gamma_{\nu} \hookrightarrow \Lambda$ of ordered groups.

Let $\mu$ be another valuation on $K[x]$ taking values in a subgroup of $\Lambda$. We say that

$$
\mu \leq \nu \quad \text { if } \quad \mu(f) \leq \nu(f), \quad \forall f \in K[x] .
$$

If moreover $\mu \neq \nu$, we write $\mu<\nu$.

Suppose that $\mu<\nu$. Let $\Phi_{\mu, \nu}$ be the set of all monic polynomials $\phi \in K[x]$ of minimal degree among those satisfying $\mu(\phi)<\nu(\phi)$.

By a well known result of MacLane-Vaquié [14, Sec. 1], any $\phi \in \Phi_{\mu, \nu}$ is a MLV key polynomial for $\mu$ and satisfies

$$
\mu(f)=\nu(f) \Longleftrightarrow \phi \nmid_{\mu} f, \quad \forall f \in K[x] .
$$

Actually, $\Phi_{\mu, \nu}$ is a whole class in $\operatorname{KP}(\mu) / \sim_{\mu}$. That is, $\Phi_{\mu, \nu}=[\phi]_{\mu}$, for all $\phi \in \Phi_{\mu, \nu}[7$, Cor. 2.5]. We define

$$
\operatorname{deg}\left(\Phi_{\mu, \nu}\right)=\operatorname{deg}(\phi) \quad \text { for any } \quad \phi \in \Phi_{\mu, \nu}
$$

For any chain $\mu<\eta<\nu$ of valuations, we have $\Phi_{\mu, \nu}=\Phi_{\mu, \eta}$ [7, Cor. 2.5]. In particular,

$$
\mu(f)=\nu(f) \Longleftrightarrow \mu(f)=\eta(f), \quad \forall f \in K[x] .
$$


Given a MLV key polynomial $\phi \in \operatorname{KP}(\nu)$ and an element $\gamma \in \Lambda \cup\{\infty\}$ such that $\gamma>$ $\nu(\phi)$, we may consider the augmented valuation $\nu^{\prime}=[\nu ; \phi, \gamma]$ defined using $\phi$-expansions as follows:

$$
f=\sum_{0 \leq s} a_{s} \phi^{s}, \quad \operatorname{deg}\left(a_{s}\right)<\operatorname{deg}(\phi) \Longrightarrow \nu^{\prime}(f)=\min \left\{\nu\left(a_{s}\right)+s \gamma \mid 0 \leq s\right\} .
$$

The polynomial $\phi$ is a MLV key polynomial for $\nu^{\prime}$ of minimal degree [6, Cor. 7.3].

\section{Abstract Key POLYNOMials}

Consider a valuation $\nu$ on $K[x]$.

Abstract key polynomials for $\nu$ were introduced by J. Decaup, W. Mahboub and M. Spivakovsky in [1] as an intrinsic characterization of the members of a complete set of key polynomials defined by F.J. Herrera Govantes, W. Mahboub, M.A. Olalla Acosta and M. Spivakovsky in [2].

In this section, we review some of these results. Our aim is to find exactly which MLV key polynomials of the valuations $\mu \leq \nu$ are abstract key polynomials for $\nu$, completing in this way some partial results from [1, Sec. 3].

2.1. Invariants of polynomials with respect to a given valuation. Let $\mathbb{N}$ be the set of positive integers. For any $b \in \mathbb{N}$, consider the linear differential operator $\partial_{b}$ on $K[x]$, defined by Taylor's formula:

$$
f(x+y)=\sum_{0 \leq b} \partial_{b}(f) y^{b}, \quad \forall f \in K[x],
$$

where $y$ is another indeterminate. Note that

$$
\partial_{b}\left(x^{n}\right)=\left(\begin{array}{c}
n \\
b
\end{array}\right) x^{n-b}, \quad \forall n \in \mathbb{N}
$$

if we agree that $\left(\begin{array}{l}n \\ b\end{array}\right)=0$ whenever $n<b$.

Let $f \in K[x]$ be a polynomial of positive degree. Denote

$$
\operatorname{mult}(f)=\text { least } b \in \mathbb{N} \text { such that } \partial_{b}(f) \neq 0 \text {. }
$$

Clearly, $\operatorname{mult}(f)=1$ if $\operatorname{char}(K)=0$. If $\operatorname{char}(K)=p$, then $\operatorname{mult}(f)=p^{r}$ is the largest power of $p$ such that $f$ belongs to $K\left[x^{p^{r}}\right]$.

This integer mult $(f)$ is an intrinsic datum of $f$. We are interested in some data that may be attached to $f$ in terms of the valuation $\nu$.

Definition 2.1. Let $f \in K[x] \backslash K$ such that $\nu(f)<\infty$. We define

$$
\epsilon(f)=\max \left\{\frac{\nu(f)-\nu\left(\partial_{b}(f)\right)}{b} \mid b \in \mathbb{N}\right\} \in\left(\Gamma_{\nu}\right)_{\mathbb{Q}} .
$$

If $\nu(f)=\infty$, we define $\epsilon(f)=\infty$.

In particular, if $\nu(f)<\infty$ we have

$$
\nu\left(\partial_{b}(f)\right) \geq \nu(f)-b \epsilon(f), \quad \forall b \geq 0,
$$

and we define $I(f) \subset \mathbb{N}$ to be the set of positive integers for which equality holds.

If $\nu(f)=\infty$ and $f$ is irreducible, we agree that $I(f)=\{\operatorname{mult}(f)\}$. Otherwise, the set $I(f)$ is not defined.

\section{Examples.}

- If $\operatorname{deg}(f)=1$, then $\epsilon(f)=\nu(f)$ and $I(f)=\{1\}$.

- If $a \in K^{*}$, then $\epsilon(a f)=\epsilon(f)$ and $I(a f)=I(f)$. 
- If $f$ is monic and $b=\operatorname{deg}(f)$, then $\partial_{b}(f)=1$. Hence, $\nu(f) / \operatorname{deg}(f) \leq \epsilon(f)$.

- If $b \notin[\operatorname{mult}(f), \operatorname{deg}(f)]$, then $\partial_{b}(f)=0$. Hence, $I(f) \subset[\operatorname{mult}(f), \operatorname{deg}(f)]$.

- For $a \in K$ and $f=(x-a)^{n}$, we have $\epsilon(f)=\nu(x-a)$ and

$$
\begin{array}{ll}
I(f)=[1, n] \cap \mathbb{N}, & \text { if } \operatorname{char}(k)=0, \\
I(f)=\left\{b \in[1, n] \cap \mathbb{N} \mid p \nmid\left(\begin{array}{c}
n \\
b
\end{array}\right)\right\}, & \text { if } \operatorname{char}(k)=p .
\end{array}
$$

Novacoski and Spivakovsky found an interesting interpretation of $\epsilon(f)$ in [10].

Proposition 2.2. Let $f \in K[x]$ be a monic polynomial such that $\nu(f)<\infty$. Let $\mathrm{Z}(f) \subset \bar{K}$ be the multiset of roots of $f$ in an algebraic closure of $K$.

For any extension $\bar{\nu}$ of $\nu$ to $\bar{K}[x]$, we have

$$
\epsilon(f)=\max \{\bar{\nu}(x-\theta) \mid \theta \in \mathrm{Z}(f)\} .
$$

Moreover, the multiplicity of $\epsilon(f)$ in the multiset $\{\bar{\nu}(x-\theta) \mid \theta \in \mathrm{Z}(f)\}$ is equal to $\max (I(f))$.

Proof. The equality (6) is proved in [10, Prop. 3.1]. We reproduce the proof because we need it to prove the second statement.

Let $\mathrm{Z}(f)=\left\{\theta_{1}, \ldots, \theta_{n}\right\}, \delta=\max \left\{\bar{\nu}\left(x-\theta_{i}\right) \mid 1 \leq i \leq n\right\}$. Let $r$ be the multiplicity of $\delta$ in this multiset. For any integer $1 \leq s \leq n$, we have

$$
\partial_{s}(f)=\sum_{J}\left(\prod_{i \notin J}\left(x-\theta_{i}\right)\right) \Longrightarrow \bar{\nu}\left(\partial_{s}(f)\right) \geq \min _{J}\left\{\sum_{i \notin J} \bar{\nu}\left(x-\theta_{i}\right)\right\},
$$

where $J$ runs on all subsets of $[1, n] \cap \mathbb{N}$ of cardinality $s$.

For $s=r$, the set $J_{0}=\left\{i \in[1, n] \cap \mathbb{N} \mid \bar{\nu}\left(x-\theta_{i}\right)=\delta\right\}$ is the unique subset of cardinality $r$ for which the term $\sum_{i \notin J_{0}} \bar{\nu}\left(x-\theta_{i}\right)$ takes the minimal value. Hence,

$$
\nu\left(\partial_{r}(f)\right)=\sum_{i \notin J_{0}} \bar{\nu}\left(x-\theta_{i}\right) .
$$

This implies

$$
\nu(f)-\nu\left(\partial_{r}(f)\right)=\sum_{i \in J_{0}} \bar{\nu}\left(x-\theta_{i}\right)=r \delta .
$$

For any $s \neq r$, let $J$ be one of the subsets of cardinality $s$ for which $\sum_{i \notin J} \bar{\nu}\left(x-\theta_{i}\right)$ takes the minimal value. Then,

$$
\nu(f)-\nu\left(\partial_{s}(f)\right) \leq \sum_{i \in J} \bar{\nu}\left(x-\theta_{i}\right) \leq s \delta
$$

This proves that $\epsilon(f)=\delta$ and $r$ belongs to $I(f)$.

Now, if $s>r$, there is at least one index $i \in J$ for which $\bar{\nu}\left(x-\theta_{i}\right)<\delta$. Hence, we get an strict inequality in (7). This proves that $s \notin I(f)$.

Corollary 2.3. For any two $f, g \in K[x] \backslash K$, we have

$$
\epsilon(f g)=\max \{\epsilon(f), \epsilon(g)\} .
$$

Moreover, if $\epsilon(f)<\epsilon(g)<\infty$, then $I(f g)=I(g)$.

Proof. The equality (8) follows immediately from Proposition 2.2.

Suppose $\epsilon(f)<\epsilon(g)<\infty$, and denote $\epsilon=\epsilon(g)=\epsilon(f g)$. For any $b \in \mathbb{N}$,

$$
\partial_{b}(f g)=\sum_{j=0}^{b} \partial_{j}(f) \partial_{b-j}(g) \Longrightarrow \nu\left(\partial_{b}(f g)\right) \geq \min \left\{\nu\left(\partial_{j}(f)\right)+\nu\left(\partial_{b-j}(g)\right) \mid 0 \leq j \leq b\right\} \text {. }
$$


For any index $j>0$ the inequality (5) shows that

$$
\nu\left(\partial_{j}(f)\right)+\nu\left(\partial_{b-j}(g)\right) \geq \nu(f)-j \epsilon(f)+\nu(g)-(b-j) \epsilon>\nu(f g)-b \epsilon .
$$

For the index $j=0$,

$$
\nu(f)+\nu\left(\partial_{b}(g)\right) \geq \nu(f)+\nu(g)-b \epsilon=\nu(f g)-b \epsilon,
$$

and equality holds if and only if $b \in I(g)$. This proves that $I(f g)=I(g)$.

Remark 2.4. If $\operatorname{supp}(\nu)=f K[x]$, then Proposition 2.2 still holds for $f$. In fact, there must be a root $\theta \in \mathrm{Z}(f)$ such that $\bar{\nu}(x-\theta)=\infty$. Then, necessarily $\operatorname{supp}(\bar{\nu})=(x-\theta) \bar{K}[x]$. Hence, the multiplicity of $\infty$ in the multiset $\{\bar{\nu}(x-\theta) \mid \theta \in \mathrm{Z}(f)\}$ is equal to the multiplicity of $\theta$ in the multiset $Z(f)$, which coincides with mult $(f)$ because $f$ is irreducible.

2.2. Abstract key polynomials. Basic properties. Following the criterion of [9], we drop the adjective "abstract" and talk simply of key polynomials for the valuation $\nu$.

Definition 2.5. A monic $Q \in K[x]$ is a key polynomial for $\nu$ if for all $f \in K[x]$, it satisfies

$$
0<\operatorname{deg}(f)<\operatorname{deg}(Q) \Longrightarrow \epsilon(f)<\epsilon(Q) \text {. }
$$

\section{Examples}

- All monic polynomials of degree one are key polynomials for $\nu$.

- If $\operatorname{supp}(\nu)=\phi K[x]$ for a monic $\phi \in K[x]$, then $\phi$ is a key polynomial for $\nu$.

On the other hand, we saw in section 1.2 that $\operatorname{KP}(\nu)=\emptyset$ in this case.

By Corollary 2.3, all key polynomials are irreducible in $K[x]$.

Let $p$ be the characteristic exponent of the valued field $(K, v)$. That is,

$$
p= \begin{cases}\operatorname{char}(k), & \text { if } \operatorname{char}(k)>0, \\ 1, & \text { if } \operatorname{char}(k)=0 .\end{cases}
$$

Proposition 2.6. [9, Prop. 2.4] If $Q \in K[x]$ is a key polynomial, then all the elements in $I(Q)$ are a power of the characteristic exponent $p$.

The next basic property of key polynomials is a generalization of [1, Prop. 10].

Lemma 2.7. Let $Q \in K[x]$ be a key polynomial for $\nu$, and let $f \in K[x]$ be non-constant polynomial such that $\epsilon(f)<\epsilon(Q)$. Consider the division with remainder in $K[x]$ :

$$
f=a+q Q, \quad \operatorname{deg}(a)<\operatorname{deg}(Q) .
$$

Then, $\nu(f)=\nu(a)<\nu(q Q)$.

Proof. Suppose that $\nu(q Q) \leq \nu(a)$. Then, we have $\nu(q Q) \leq \nu(f)$ as well. Let us show that this leads to a contradiction.

By Corollary 2.3, $a \neq 0$. Let us agree that $\epsilon(a)=-\infty$ if $a \in K^{*}$.

Since $Q$ is a key polynomial, $\epsilon(a)<\epsilon(Q)$. For any $b \in I(q Q)$, we have

$$
\begin{gathered}
\nu\left(\partial_{b}(f)\right) \geq \nu(f)-b \epsilon(f)>\nu(f)-b \epsilon(Q) \geq \nu(q Q)-b \epsilon(Q), \\
\nu\left(\partial_{b}(a)\right) \geq \nu(a)-b \epsilon(a)>\nu(a)-b \epsilon(Q) \geq \nu(q Q)-b \epsilon(Q) .
\end{gathered}
$$

Since $\partial_{b}(q Q)=\partial_{b}(f)-\partial_{b}(a)$, we deduce

$$
\nu(q Q)-b \epsilon(q Q)=\nu\left(\partial_{b}(q Q)\right)>\nu(q Q)-b \epsilon(Q) .
$$

This implies $\epsilon(q Q)<\epsilon(Q)$, contradicting Corollary 2.3.

Corollary 2.8. Let $Q \in K[x]$ be a key polynomial for $\nu$, and let $f \in K[x]$ be non-constant polynomial such that $\epsilon(f)<\epsilon(Q)$. Then, $\operatorname{in}_{\nu} f$ is a unit in $\mathcal{G}_{\nu}$. 
Proof. Corollary 2.3 shows that $f$ is not divisible by $Q$ in $K[x]$. Since $Q$ is irreducible, there is a Bézout identity in $K[x]$ :

$$
a Q+b f=1, \quad \operatorname{deg}(b)<\operatorname{deg}(Q) .
$$

By Lemma 2.7, $b f \sim_{\nu} 1$, or equivalently, $\left(\operatorname{in}_{\nu} b\right)\left(\operatorname{in}_{\nu} f\right)=\operatorname{in}_{\nu} 1$ in $\mathcal{G}_{\nu}$.

Proposition 2.9. [1, Prop. 12] If $Q$ is a key polynomial for $\nu$, then the $\nu$-truncation function $\nu_{Q}$ is a valuation on $K[x]$ such that $\nu_{Q} \leq \nu$.

2.3. Comparison between abstract and MLV key polynomials. If $\nu$ has trivial support, then any key polynomial $Q$ for $\nu$ is a MLV key polynomial for $\nu_{Q}[1$, Thm. 23].

The following result does not assume trivial support and it shows that $Q$ has minimal degree in $\operatorname{KP}\left(\nu_{Q}\right)$.

Proposition 2.10. If $Q$ is a key polynomial for $\nu$ such that $\nu(Q)<\infty$, then $Q$ is a $M L V$ key polynomial of minimal degree for $\nu_{Q}$.

Proof. By Lemma 1.2, $Q$ is $\nu_{Q}$-minimal. Thus, $Q \nmid_{\nu} 1$, so that $\operatorname{in}_{\nu} Q$ is not a unit in $\mathcal{G}_{\nu}$.

On the other hand, for all polynomials $f \in K[x]$ of degree less than $\operatorname{deg}(Q)$, the element $\operatorname{in}_{\nu} f$ is a unit in $\mathcal{G}_{\nu}$. In fact, this follows from $\epsilon(f)<\epsilon(Q)$, by Corollary 2.8.

Hence, $Q$ is a MLV key polynomial of minimal degree for $\nu_{Q}$ [6, Thm. 3.2+Prop. 3.5].

The rest of the section is devoted to analyzing which MLV polynomials of valuations $\mu \leq \nu$ are (abstract) key polynomials for $\nu$. The next two results are crucial for this purpose.

Proposition 2.11. [1, Lem. 14+Prop. 15], [9, Prop. 2.7] Let $Q \in K[x]$ be a key polynomial for $\nu$. Let $f=\sum_{0 \leq s} a_{s} Q^{s}$ be the canonical $Q$-expansion of a non-zero $f \in K[x]$. Denote by $S_{\nu, Q}(f)$ the set of indices $s$ for which $\nu\left(a_{s} Q^{s}\right)=\nu_{Q}(f)$. Then,

(i) For all $b \in \mathbb{N}$ we have

$$
\nu_{Q}\left(\partial_{b}(f)\right) \geq \nu_{Q}(f)-b \epsilon(Q) .
$$

(ii) If $S_{\nu, Q}(f) \neq\{0\}$, then equality holds in (9) for some $b \in \mathbb{N}$.

(iii) If equality holds in (9) for $b \in \mathbb{N}$ and $\nu_{Q}\left(\partial_{b}(f)\right)=\nu\left(\partial_{b}(f)\right)$, then $\epsilon(f) \geq \epsilon(Q)$. If in addition, $\nu(f)>\nu_{Q}(f)$, then $\epsilon(f)>\epsilon(Q)$.

Proposition 2.12. [1, Prop. 20+Lem. 24] Let $Q, Q^{\prime} \in K[x]$ be key polynomials for $\nu$. Then,

$$
\epsilon(Q) \leq \epsilon\left(Q^{\prime}\right) \Longleftrightarrow \nu_{Q} \leq \nu_{Q^{\prime}}
$$

In this case, $\nu_{Q^{\prime}}(Q)=\nu(Q)$. Moreover, $\epsilon(Q)<\epsilon\left(Q^{\prime}\right)$ if and only if $\nu_{Q}\left(Q^{\prime}\right)<\nu\left(Q^{\prime}\right)$.

From now on, we fix a valuation $\mu$ on $K[x]$ with values in the group $\Gamma_{\nu}$ and satisfying

$$
\mu \leq \nu
$$

Let us first determine for which MLV key polynomials $\phi$ for $\mu$ the truncation $\nu_{\phi}$ is a valuation.

Lemma 2.13. Suppose $\mu<\nu$, and take $\phi \in \mathrm{KP}(\mu)$.

(1) $\phi \in \Phi_{\mu, \nu} \Longrightarrow \nu_{\phi}$ is a valuation and $\mu<\nu_{\phi} \leq \nu$.

(2) $\phi \notin \Phi_{\mu, \nu}, \operatorname{deg}(\phi) \leq \operatorname{deg}\left(\Phi_{\mu, \nu}\right) \Longrightarrow \nu_{\phi}=\mu$.

(3) $\operatorname{deg}(\phi)>\operatorname{deg}\left(\Phi_{\mu, \nu}\right) \Longrightarrow \nu_{\phi}$ is not a valuation. 
Proof. (1) For any $\phi \in \Phi_{\mu, \nu}$, the function $\nu_{\phi}$ coincides with the augmented valuation $[\mu ; \phi, \nu(\phi)]$ introduced in section 1.3. The inequalities $\mu<\nu_{\phi} \leq \nu$ are obvious.

(2) Suppose $\phi \notin \Phi_{\mu, \nu}$ and $\operatorname{deg}(\phi) \leq \operatorname{deg}\left(\Phi_{\mu, \nu}\right)$. Then,

$$
\mu(\phi)=\nu(\phi), \quad \mu(a)=\nu(a), \forall a \in K[x] \text { with } \operatorname{deg}(a)<\operatorname{deg}(\phi) .
$$

Hence, $\mu_{\phi}=\nu_{\phi}$. Since $\phi$ is $\mu$-minimal, Lemma 1.2 shows that $\mu=\mu_{\phi}=\nu_{\phi}$.

(3) By section 1.3, $\Phi_{\mu, \nu}=[Q]_{\mu}$ for some $Q \in \operatorname{KP}(\mu)$. Suppose that $\operatorname{deg}(\phi)>\operatorname{deg}(Q)$ and $\nu_{\phi}$ is a valuation. Let us derive a contradiction.

By the definition of $\nu_{\phi}$ and Lemma 1.2, $\phi$ is $\nu_{\phi}$-minimal. Hence, $\operatorname{in}_{\nu_{\phi}} \phi$ is not a unit in $\mathcal{G}_{\nu_{\phi}}$. By Theorem 1.4, the set $\operatorname{KP}\left(\nu_{\phi}\right)$ is not empty.

Therefore, we may apply [6, Thm. 3.9] to both valuations $\mu$ and $\nu_{\phi}$. For all monic polynomials $f \in K[x]$ we have

$$
\frac{\mu(f)}{\operatorname{deg}(f)} \leq \frac{\mu(\phi)}{\operatorname{deg}(\phi)}, \quad \frac{\nu_{\phi}(f)}{\operatorname{deg}(f)} \leq \frac{\nu_{\phi}(\phi)}{\operatorname{deg}(\phi)},
$$

and equality holds if and only if $f$ is $\mu$-minimal, or $\nu_{\phi}$-minimal, respectively.

If we apply these inequalities to $f=Q$ we get a contradiction:

$$
\frac{\mu(Q)}{\operatorname{deg}(Q)}<\frac{\nu(Q)}{\operatorname{deg}(Q)}=\frac{\nu_{\phi}(Q)}{\operatorname{deg}(Q)} \leq \frac{\nu_{\phi}(\phi)}{\operatorname{deg}(\phi)}=\frac{\nu(\phi)}{\operatorname{deg}(\phi)}=\frac{\mu(\phi)}{\operatorname{deg}(\phi)}=\frac{\mu(Q)}{\operatorname{deg}(Q)}
$$

where the last equality holds because $Q$ is $\mu$-minimal.

Lemma 2.14. [9, Lem. 2.11] Let $Q$ be a key polynomial for $\nu$ such that $\nu_{Q}<\nu$. Then, all the polynomials in $\Phi_{\nu_{Q}, \nu}$ are key polynomials for $\nu$.

Proposition 2.15. Let $\mu$ be a valuation on $K[x]$ such that $\mu \leq \nu$. Then all the $M L V$ key polynomials for $\mu$ of minimal degree are key polynomials for $\nu$.

Proof. If $\mu=\nu$ and $\operatorname{KP}(\nu)=\emptyset$, the statement of the Proposition is vacuously true. Therefore, in the case $\mu=\nu$ we may assume that $\operatorname{KP}(\nu) \neq \emptyset$,

We proceed by induction on $\operatorname{deg}(\mu)$. If $\operatorname{deg}(\mu)=1$, the statement is obvious because all the monic polynomials of degree one are key polynomials.

Suppose $\operatorname{deg}(\mu) \geq 2$ and the statement holds for all valuations $\rho<\nu$ of degree less than $\operatorname{deg}(\mu)$. Let $\phi \in \operatorname{KP}(\mu)$ be a MLV key polynomial for $\mu$ of minimal degree $\operatorname{deg}(\phi)=\operatorname{deg}(\mu)$.

Since $\operatorname{KP}(\mu) \neq \emptyset,[7$, Lem. 4.5] shows that $\mu$ has finite depth. By the theorem of MacLane-Vaquié, $\mu$ is the augmentation of a valuation $\rho$ of smaller degree. Let us discuss in an independent way the cases in which $\mu$ is an ordinary or a limit augmentation of $\rho$.

Ordinary augmentation. We have $\mu=[\rho ; \chi, \mu(\chi)]$, for a certain MLV key polynomial $\chi \in \operatorname{KP}(\rho)$ satisfying $\mu(\chi)>\rho(\chi)$, which becomes a MLV key polynomial of minimal degree for $\mu$ [6, Cor. 7.3].

In particular, $\operatorname{deg}(\phi)=\operatorname{deg}(\chi)$ and $\mu(\phi)=\mu(\chi)$ [6, Thm. 3.9]. Let us write $\phi=\chi+a$, with $a \in K[x]$ of degree less than $\operatorname{deg}(\chi)$. Since $\Phi_{\rho, \mu}=[\chi]_{\rho}$, we have

$$
\rho(a)=\mu(a) \geq \mu(\chi)>\rho(\chi) .
$$

Hence, $\phi \sim_{\rho} \chi$, so that $\phi \in \Phi_{\rho, \mu}$.

Now, let $Q \in \operatorname{KP}(\rho)$ be a MLV key polynomial for $\rho$ of minimal degree; that is, $\operatorname{deg}(Q)=$ $\operatorname{deg}(\rho)<\operatorname{deg} \mu)=\operatorname{deg}(\phi)$. By the induction hypothesis, $Q$ is a key polynomial for $\nu$.

Since $\phi \nmid_{\rho} Q$, we have $Q \notin[\phi]_{\rho}=\Phi_{\rho, \mu}=\Phi_{\rho, \nu}$. By Lemma 2.13, $\nu_{Q}=\rho$. Thus, Lemma 2.14 shows that $\phi \in \Phi_{\rho, \mu}$ is a key polynomial for $\nu$. 
Limit augmentation. The valuation $\rho$ admits a well-ordered set $\left(\rho_{\alpha}\right)_{\alpha<\lambda}$ of ordinary augmentations of constant degree $m$, determined by MLV key polynomials $\chi_{\alpha} \in \operatorname{KP}(\rho)$ of degree $m$ such that $\rho_{\alpha}=\mu_{\chi_{\alpha}}=\nu_{\chi_{\alpha}}$.

All the polynomials $f \in K[x]$ of degree less than or equal to $m$ are stable; that is, $\rho_{\alpha}(f)=\mu(f)=\nu(f)$ for all $\alpha$ sufficiently large.

There are polynomials which are not stable, and we have $\mu=\nu_{\varphi}$ for some monic unstable $\varphi \in K[x]$ of minimal degree, which becomes a MLV key polynomial of minimal degree for $\mu$ [6, Cor. 7.13]. See section 3.1 for a more precise definition of limit augmentations.

In particular, $\operatorname{deg}(\varphi)=\operatorname{deg}(\phi)$ and $\mu(\varphi)=\mu(\phi)[6$, Thm. 3.9]. Let us write $\phi=\varphi+a$, with $a \in K[x]$ of degree less than $\operatorname{deg}(\varphi)$. By the minimality of $\operatorname{deg}(\varphi)$, the polynomial $a$ is stable; that is, for some index $\alpha_{0}$ we have

$$
\rho_{\alpha}(a)=\mu(a) \geq \mu(\varphi)>\rho_{\alpha}(\varphi), \quad \forall \alpha \geq \alpha_{0} .
$$

Hence, $\phi \sim_{\rho_{\alpha}} \varphi$ for all $\alpha \geq \alpha_{0}$. This implies that $\phi$ is unstable too:

$$
\rho_{\alpha}(\phi)=\rho_{\alpha}(\varphi)<\mu(\varphi)=\mu(\phi), \quad \forall \alpha \geq \alpha_{0} .
$$

By the induction hypothesis, all the $\chi_{\alpha}$ are key polynomials for $\nu$. Take any $b \in$ $[1, \operatorname{deg}(\phi)] \cap \mathbb{N}$. Since $\operatorname{deg}\left(\partial_{b}(\phi)\right)<\operatorname{deg}(\phi)$, the polynomial $\partial_{b}(\phi)$ is stable. Take $\alpha$ sufficiently large so that

$$
\rho_{\alpha}\left(\partial_{b}(\phi)\right)=\mu\left(\partial_{b}(\phi)\right)=\nu\left(\partial_{b}(\phi)\right), \quad \forall b \in \mathbb{N} .
$$

By [2, Sec. 4], or [13, Sec. 3], the integers $\max \left(S_{\nu, \chi_{\alpha}}(\phi)\right)$ are all positive, and stabilize for a sufficiently large index $\alpha$. In particular, $S_{\nu, \chi_{\alpha}}(\phi) \neq\{0\}$ for all $\alpha$. By (iii) of Proposition 2.11, $\epsilon(\phi)>\epsilon\left(\chi_{\alpha}\right)$ for all $\alpha$ sufficiently large.

Now, take any $f \in K[x]$ with $\operatorname{deg}(f)<\operatorname{deg}(\phi)$. Since $f$ and $\partial_{b}(f)$ are stable, we may take $\alpha$ sufficiently large so that

$$
\rho_{\alpha}(f)=\nu(f), \quad \rho_{\alpha}\left(\partial_{b}(f)\right)=\nu\left(\partial_{b}(f)\right), \quad \forall b \in \mathbb{N} .
$$

By (i) of Proposition 2.11, for all $b \in \mathbb{N}$ we have

$$
\frac{\nu(f)-\nu\left(\partial_{b}(f)\right)}{b}=\frac{\rho_{\alpha}(f)-\rho_{\alpha}\left(\partial_{b}(f)\right)}{b} \leq \epsilon\left(\chi_{\alpha}\right)<\epsilon(\phi) .
$$

Thus, $\phi$ is a key polynomial for $\nu$.

Lemma 2.13 exhibited some MLV key polynomials for $\mu$ that are not (abstract) key polynomials for $\nu$. The next lemma offers some more examples.

Lemma 2.16. Suppose $\mu<\nu$, and take $\phi \in \operatorname{KP}(\mu)$. If $\phi \notin \Phi_{\mu, \nu}$ and $\operatorname{deg}(\phi)>\operatorname{deg}(\mu)$, then $\phi$ is not a key polynomial for $\nu$.

Proof. If $\operatorname{deg}(\phi)>\operatorname{deg}\left(\Phi_{\mu, \nu}\right)$, the Lemma follows from Lemma 2.13 and Proposition 2.9. Suppose $\operatorname{deg}(\mu)<\operatorname{deg}(\phi) \leq \operatorname{deg}\left(\Phi_{\mu, \nu}\right)$.

Let $\phi_{0}$ be a MLV key polynomial of minimal degree $\operatorname{deg}\left(\phi_{0}\right)=\operatorname{deg}(\mu)$. By Lemma 2.13, $\nu_{\phi}=\mu=\nu_{\phi_{0}}$.

By Proposition $2.15, \phi_{0}$ is a key polynomial for $\mu$. Hence, $\phi$ cannot be a key polynomial because it would satisfy $\epsilon(\phi)>\epsilon\left(\phi_{0}\right)$, contradicting Proposition 2.12 .

We may summarize the results obtained so far in the next two theorems.

Theorem 2.17. Suppose that $\mu<\nu$ and $\phi \in \operatorname{KP}(\mu)$. Then, $\phi$ is a key polynomial for $\nu$ if and only if it satisfies one of the following two conditions.

(1) $\phi \in \Phi_{\mu, \nu}$

(2) $\phi \notin \Phi_{\mu, \nu}$ and $\operatorname{deg}(\phi)=\operatorname{deg}(\mu)$.

In the first case, $\nu_{\phi}=[\mu ; \phi, \nu(\phi)]$. In the second case, $\nu_{\phi}=\mu$. 
Theorem 2.18. Let $\phi \in \operatorname{KP}(\nu)$. Then, $\phi$ is a key polynomial for $\nu$ if and only if $\operatorname{deg}(\phi)=$ $\operatorname{deg}(\nu)$. In this case, $\nu_{\phi}=\nu$.

By Theorem 1.7, two $\mu$-equivalent MLV key polynomials for $\mu$ have the same degree. Hence, the next result follows immediately from Theorems 2.17 and 2.18.

Corollary 2.19. Suppose that $\mu \leq \nu$ and $\phi \in \operatorname{KP}(\mu)$. If $\phi$ is a key polynomial for $\nu$, then all the polynomials in $[\phi]_{\mu}$ are key polynomials for $\nu$ too.

Corollary 2.20. Let $\phi \in \operatorname{KP}(\nu)$ of minimal degree. Then, $\epsilon(\phi) \geq \epsilon(f)$ for all $f \in K[x]$.

Proof. By Theorem 2.18, $\phi$ is a key polynomial for $\nu$ and $\nu_{\phi}=\nu$.

The result follows from (i) of Proposition 2.11.

Also, these results lead to another characterization of abstract key polynomials.

Theorem 2.21. Let $\nu$ be a valuation on $K[x]$, and $Q \in K[x]$ a monic polynomial. The following conditions are equivalent.

(1) $Q$ is a key polynomial for $\nu$.

(2) $\nu_{Q}$ is a valuation and either $\operatorname{supp}(\nu)=Q K[x]$, or $Q$ is a $M L V$ key polynomial for $\nu_{Q}$ of minimal degree.

(3) $\nu_{Q}$ is a valuation and $Q$ has minimal degree among all the monic polynomials $f \in$ $K[x]$ satisfying $\nu_{f}=\nu_{Q}$.

Proof. $(1) \Rightarrow(2)$ follows from Propositions 2.9 and 2.10 .

$(2) \Rightarrow(1)$ follows from Proposition 2.15.

$(2) \Rightarrow(3)$. Let $f \in K[x]$ be a monic polynomial such that $\nu_{f}=\nu_{Q}$. If $\operatorname{supp}(\nu)=Q K[x]$, then $\nu(f)=\nu_{f}(f)=\nu_{Q}(f)=\infty$, so that $f$ is a multiple of $Q$.

Suppose that $Q$ is a MLV key polynomial for $\nu_{Q}$ of minimal degree. By Lemma 1.2, $f$ is $\nu_{Q}$-minimal; thus, $\operatorname{deg}(f)$ is a multiple of $\operatorname{deg}(Q)$ [6, Prop. 3.7].

$(3) \Rightarrow(2)$. Suppose $\operatorname{supp}(\nu) \neq Q K[x]$. Then, (3) implies that $\nu(Q)<\infty$. By Lemma $1.2, Q$ has minimal degree among all the $\nu_{Q}$-minimal polynomials.

Let $Q_{0}$ be a MLV key polynomial for $\nu_{Q}$ of minimal degree. By [6, Prop. 3.7], $Q=Q_{0}+a$ for some $a \in K[x]$ with $\operatorname{deg}(a)<\operatorname{deg}\left(Q_{0}\right)$ and $\nu_{Q}(a) \geq \nu_{Q}\left(Q_{0}\right)$. Hence, either $Q \sim_{\nu_{Q}} Q_{0}$ (if $\nu_{Q}(a)>\nu_{Q}\left(Q_{0}\right)$ ), or $\operatorname{deg}(R(Q))=1$ (if $\nu_{Q}(a)=\nu_{Q}\left(Q_{0}\right)$ ). By Theorem 1.7, $Q$ is a MLV key polynomial for $\nu_{Q}$ of minimal degree.

A key polynomial $Q$ for $\nu$ is said to be maximal if $\nu_{Q}=\nu$. These key polynomials admit the following characterization.

Corollary 2.22. Let $\nu$ be a valuation on $K[x]$, and $Q \in K[x]$ a monic polynomial. The following conditions are equivalent.

(1) $Q$ is a maximal key polynomial for $\nu$.

(2) Either $\operatorname{supp}(\nu)=Q K[x]$, or $Q$ is a $M L V$ key polynomial for $\nu$ of minimal degree.

(3) $\epsilon(Q) \geq \epsilon(f)$ for all the polynomials $f \in K[x]$, and $Q$ has minimal degree among all the polynomials with this property.

Proof. Theorem 2.21 shows that (1) and (2) are equivalent.

$(2) \Rightarrow(3)$. Corollary 2.20 shows that $\epsilon(Q) \geq \epsilon(f)$ for all the polynomials $f \in K[x]$.

Since $Q$ is a key polynomial for $\nu$, for any polynomial $f$ of smaller degree $\epsilon(f)$ cannot be maximal because $\epsilon(f)<\epsilon(Q)$.

$(3) \Rightarrow(1)$. By definition, a monic polynomial of minimal degree for which $\epsilon(Q)$ takes a maximal value is a key polynomial for $\nu$. Finally, $\nu_{Q}=\nu$ by Proposition 2.12.

Only the valuations $\nu$ of finite depth admit maximal key polynomials. 


\section{Comparison of Maclane-Vaquié and abstract limit key polynomials}

3.1. MacLane-Vaquié limit key polynomials. Let us recall the definition of MacLaneVaquié (MLV) limit key polynomials [14, Sec. 1.4].

Let $\mu$ be a valuation on $K[x]$ admitting MLV key polynomials.

Definition 3.1. A continuous family of augmentations of $\mu$ is a family

$$
\left(\rho_{i}=\left[\mu ; \chi_{i}, \gamma_{i}\right]\right)_{i \in A}
$$

of ordinary augmentations of $\mu$, parameterized by a set $A$, satisfying the following conditions:

(1) The set $A$ is totally ordered and contains no maximal element.

(2) All $M L V$ key polynomials $\chi_{i} \in \operatorname{KP}(\mu)$ have the same degree.

(3) For all $i<j$ in $A, \chi_{j}$ is a $M L V$ key polynomial for $\rho_{i}$ and satisfies

$$
\chi_{j} \chi_{\rho_{i}} \chi_{i} \text { and } \rho_{j}=\left[\rho_{i} ; \chi_{j}, \gamma_{j}\right] \text {. }
$$

The common degree $m=\operatorname{deg}\left(\chi_{i}\right)$, for all $i$, is called the stable degree of the family.

The basic example of a continuous family of augmentations is provided by any valuation $\nu$ on $K[x]$ such that $\mu<\nu$.

Proposition 3.2. Let $\nu$ be a valuation on $K[x]$ such that $\mu<\nu$. Suppose that the set $A=\nu\left(\Phi_{\mu, \nu}\right)$ does not contain a maximal element. For any $\alpha \in A$, choose any polynomial $\chi_{\alpha} \in \Phi_{\mu, \nu}$ such that $\nu\left(\chi_{\alpha}\right)=\alpha$, and build $\rho_{\alpha}=\left[\mu ; \chi_{\alpha}, \alpha\right]=\nu_{\chi_{\alpha}}$. Then, $\left(\rho_{\alpha}\right)_{\alpha \in A}$ is a continuous family of augmentations of $\mu$.

Proof. Clearly, the family $\left(\rho_{\alpha}\right)_{\alpha \in A}$ satisfies conditions (1) and (2) of Definition 3.1.

Let $m=\operatorname{deg}\left(\Phi_{\mu, \nu}\right)$. For $\alpha<\beta$ in $A$, write $\chi_{\beta}=\chi_{\alpha}+a$ for some $a \in K[x]$ of degree less than $m$. Since $\nu\left(\chi_{\alpha}\right)=\alpha<\beta=\nu\left(\chi_{\beta}\right)$, we deduce that $\mu(a)=\nu(a)=\alpha$. By the definition of the augmented valuations,

$$
\rho_{\alpha}\left(\chi_{\beta}\right)=\alpha<\beta=\rho_{\beta}\left(\chi_{\beta}\right), \quad \rho_{\alpha}\left(\chi_{\alpha}\right)=\alpha=\rho_{\beta}\left(\chi_{\alpha}\right) .
$$

As we saw in section 1.3, these equations show that $\chi_{\alpha} \notin \Phi_{\rho_{\alpha}, \rho_{\beta}}=\left[\chi_{\beta}\right]_{\rho_{\alpha}}$. In particular, $\chi_{\beta}$ is a MLV key polynomial for $\rho_{\alpha}$ and $\chi_{\alpha} \chi_{\rho_{\alpha}} \chi_{\beta}$.

Finally, $\left[\rho_{\alpha} ; \chi_{\beta}, \beta\right]=\rho_{\beta}$, because both valuations coincide on $\chi_{\beta}$-expansions.

A polynomial $f \in K[x]$ is stable with respect to the family $\left(\rho_{i}\right)_{i \in A}$ if

$$
\rho_{i}(f)=\rho_{i_{0}}(f), \quad \forall i \geq i_{0},
$$

for some index $i_{0}$. This stable value is denoted by $\rho_{\infty}(f)$.

By the equivalence (4), an unstable polynomial $f$ satisfies necessarily

$$
\rho_{i}(f)<\rho_{j}(f), \quad \forall i<j .
$$

Let $m_{\infty}$ be the minimal degree of an unstable polynomial. We agree that $m_{\infty}=\infty$ if all polynomials are stable. The following properties hold for all continuous families:

- The mappings defined by $i \mapsto \gamma_{i}$ and $i \mapsto \rho_{i}$ are isomorphisms of ordered sets between $A$ and $\left\{\gamma_{i} \mid i \in A\right\},\left\{\rho_{i} \mid i \in A\right\}$, respectively.

- For all $i \in A, \chi_{i}$ is a MLV key polynomial for $\rho_{i}$, of minimal degree.

- For all $i \in A, \operatorname{deg}\left(\rho_{i}\right)=m \leq m_{\infty}$.

- For all $i, j \in A, \rho_{i}\left(\chi_{j}\right)=\min \left\{\gamma_{i}, \gamma_{j}\right\}$. In particular, all the polynomials $\chi_{i}$ are stable.

- $\Phi_{\rho_{i}, \rho_{j}}=\left[\chi_{j}\right]_{\rho_{i}}$ for all $i<j$ in $A$.

- All the valuations $\rho_{i}$ are residually transcendental.

- If $i$ is not a minimal element in $A$ then $\rho_{i}$ has relative ramification index equal to one (cf. Definition 1.3). In particular, all the value groups $\Gamma_{\rho_{i}}$ coincide. 
The common value grup $\Gamma_{\infty}:=\Gamma_{\rho_{i}}$ for all $i$, is called the stable value group of the continuous family. Note that $\gamma_{i} \in \Gamma_{\infty}$ for all $i$.

Remark. Any cofinal family of $\left(\rho_{i}\right)_{i \in A}$ will have the same limit behaviour. Since all totally ordered sets admit well-ordered cofinal subsets, we may always assume that the set $A$ is well-ordered.

Essential continuous families of augmentations. Any continuous family falls in one of the following three cases:

(a) It has a stable limit. That is, $m_{\infty}=\infty$, so that the function $\rho_{\infty}$ is a valuation on $K[x]$. This valuation is commensurable and satisfies $\operatorname{KP}\left(\rho_{\infty}\right)=\emptyset$.

(b) It is inessential. That is, $m_{\infty}=m$.

(c) It is essential. That is, $m<m_{\infty}<\infty$.

Let $\nu$ be a valuation on $K[x]$ such that $\rho_{i}<\nu$ for all $i \in A$.

If $\left(\rho_{i}\right)_{i \in A}$ is inessential and $f \in K[x]$ is an unstable polynomial of degree $m$, then the ordinary augmentation $\mu=[\rho ; f, \nu(f)]$ satisfies

$$
\rho_{i}<\mu \leq \nu, \quad \forall i \in A .
$$

In other words, $\mu$ is closer to $\nu$ than any $\rho_{i}$, and $\mu$ is obtained from $\rho$ by a single augmentation. In the terminology of [7], we may avoid the continuous family $\left(\rho_{i}\right)_{i \in A}$ along the process of constructing a MacLane-Vaquié chain of valuations for $\nu$.

In the terminology of [2], all the key polynomials $\chi_{i}$ may be replaced by the single key polynomial $f$ in any complete set of key polynomials for $\mu$.

This justifies why we call it "inessential".

Only the essential continuous families admit (non-fake) limit key polynomials. From now on, we suppose that our chain $\left(\rho_{i}\right)_{i \in A}$ is essential.

We define the set of MLV limit key polynomials for $\left(\rho_{i}\right)_{i \in A}$ :

$$
\mathrm{KP}_{\infty}=\mathrm{KP}_{\infty}\left(\left(\rho_{i}\right)_{i \in A}\right),
$$

as the set of monic unstable polynomials in $K[x]$ of minimal degree $m_{\infty}$.

Take $\phi \in \mathrm{KP}_{\infty}$. Let $\Gamma_{\infty} \hookrightarrow \Lambda$ be an embedding of ordered groups, and choose $\gamma \in \Lambda \cup\{\infty\}$ such that

We may consider a limit augmentation

$$
\gamma>\rho_{i}(\phi), \quad \forall i \in A .
$$

$$
\mu_{\phi, \gamma}=\left[\left(\rho_{i}\right)_{i \in A} ; \phi, \gamma\right]
$$

which on $\phi$-expansions $f=\sum_{0 \leq s} a_{s} \phi^{s}$ acts as follows:

$$
\mu_{\phi, \gamma}(f)=\min \left\{\rho_{\infty}\left(a_{s}\right)+s \gamma \mid 0 \leq s\right\}=\min \left\{\mu_{\phi, \gamma}\left(a_{s} \phi^{s}\right) \mid 0 \leq s\right\} .
$$

This function $\mu_{\phi, \gamma}$ is a valuation on $K[x]$ which satisfies $\mu_{\phi, \gamma}>\rho_{i}$ for all $i \in A$.

Let $\nu$ be a valuation on $K[x]$ such that $\nu>\rho_{i}$ for all $i \in A$.

For every stable polyomial $f$ one has $\nu(f)=\rho_{\infty}(f)$. In particular,

$$
\nu\left(\chi_{i}\right)=\rho_{\infty}\left(\chi_{i}\right)=\gamma_{i}, \quad \text { for all } i \in A .
$$

Any MLV limit key polynomial $\phi \in \mathrm{KP}_{\infty}$ is a key polynomial for $\nu$.

Indeed, take $\gamma=\nu(\phi)$. For all $i<j$ in $A$, we have $\rho_{i}(\phi)<\rho_{j}(\phi) \leq \nu(\phi)=\gamma$. The limit augmented valuation $\mu_{\phi, \gamma}$ clearly satisfies $\mu_{\phi, \gamma} \leq \nu$. By [6, Cor. 7.13], $\phi$ is a MLV key polynomial for $\mu_{\phi, \gamma}$ of minimal degree. Thus, our claim follows from Proposition 2.15.

In the following section we show that $\phi$ is actually a limit key polynomial for $\nu$. 
3.2. Abstract limit key polynomials. Let $\nu$ be a valuation on $K[x]$. Novacoski and Spivakovsky define in [9] an (abstract) limit key polynomial for $\nu$ as a monic polynomial $Q \in K[x]$ for which there exists a key polynomial $Q_{-}$satisfying the following conditions.

(K1) $\operatorname{deg}\left(Q_{-}\right)=\operatorname{deg}\left(\Phi_{\nu_{Q_{-}}, \nu}\right)$.

(K2) the set $\left\{\nu(\chi) \mid \chi \in \Phi_{\nu_{Q_{-}}, \nu}\right\}$ has no maximal element.

(K3) $\nu_{\chi}(Q)<\nu(Q)$ for all $\chi \in \Phi_{\nu_{Q_{-}}, \nu}$.

(K4) $Q$ has minimal degree among all polynomials satisfying (K3).

Proposition 3.3. Let $\left(\rho_{i}\right)_{i \in A}$ be an essential continuous family of augmentations of a valuation $\mu$. Let $\nu$ be a valuation on $K[x]$ such that $\nu>\rho_{i}$ for all $i \in A$. Then, all $M L V$ limit key polynomials for $\left(\rho_{i}\right)_{i \in A}$ are limit key polynomials for $\nu$.

Proof. Take $\phi \in \mathrm{KP}_{\infty}$ and let us fix any $i \in A$. Take $Q_{-}=\chi_{i}$, which is a key polynomial by Proposition 2.15. By the arguments in section 1.3, we have

$$
\left[\chi_{j}\right]_{\rho_{i}}=\Phi_{\rho_{i}, \rho_{j}}=\Phi_{\rho_{i}, \nu}, \quad \forall j>i .
$$

Since $\chi_{i} \nmid \rho_{i} \chi_{j}$, we have $\chi_{i} \notin\left[\chi_{j}\right]_{\rho_{i}}=\Phi_{\rho_{i}, \nu}$, and Lemma 2.13 shows that $\nu_{\chi_{i}}=\rho_{i}$. Since $\operatorname{deg}\left(\chi_{i}\right)=\operatorname{deg}\left(\chi_{j}\right)=\operatorname{deg}\left(\Phi_{\rho_{i}, \nu}\right)$, condition (K1) is satisfied.

Since our continuous family is essential, all the polynomials of degree $m$ are stable. Thus, for any $\chi \in \Phi_{\nu_{Q_{-}, \nu}}=\Phi_{\rho_{i}, \nu}$ there exists $j \in A$ such that $\nu(\chi)=\rho_{\infty}(\chi)=\rho_{j}(\chi)$. By $[6$, Thm. 3.9] we deduce (K2), because

$$
\nu(\chi)=\rho_{j}(\chi) \leq \rho_{j}\left(\chi_{j}\right)=\gamma_{j}<\gamma_{k}=\nu\left(\chi_{k}\right), \text { for all } j<k .
$$

Also, the inequality $\nu(\chi)<\gamma_{k}$ implies $\nu_{\chi}<\rho_{k}$, by an obvious comparison of the two valuations on $\chi$-expansions. Hence, $\nu_{\chi}(\phi) \leq \rho_{k}(\phi)<\nu(\phi)$. This proves (K3).

Finally, any monic polynomial $Q$ satisfying (K3) is unstable, Thus, $\operatorname{deg}(Q) \geq m_{\infty}=$ $\operatorname{deg}(\phi)$. This proves $(\mathrm{K} 4)$.

The converse statement holds too.

Proposition 3.4. Let $Q \in K[x]$ be a limit key polynomial for $\nu$. Then, $Q$ is a MLV limit key polynomial for some essential continuous family of augmentations.

Proof. Let $Q_{-} \in K[x]$ be a key polynomial such that the pair $Q, Q_{-}$satisfies conditions (K1)-(K4). Define $\mu=\nu_{Q_{-}}$and $m=\operatorname{deg}\left(Q_{-}\right)$. By (K1), all polynomials in $\Phi_{\mu, \nu}$ have degree $m$.

By (K2), the totally ordered set $A:=\nu\left(\Phi_{\mu, \nu}\right)$ contains no maximal element. Consider the continuous family of augmentations $\left(\rho_{\alpha}\right)_{\alpha \in A}$ described in Proposition 3.2.

For any $\chi \in \Phi_{\mu, \nu}$, let $\alpha=\nu(\chi) \in A$. By definition, $\rho_{\alpha}=\nu_{\chi_{\alpha}}$ for some $\chi_{\alpha} \in \Phi_{\mu, \nu}$ such that $\nu\left(\chi_{\alpha}\right)=\alpha$. The valuations $\nu_{\chi}$ and $\nu_{\chi_{\alpha}}$ can be seen as augmentations of $\mu$ :

$$
\nu_{\chi_{\alpha}}=\left[\mu ; \chi_{\alpha}, \alpha\right], \quad \nu_{\chi}=[\mu ; \chi, \alpha] .
$$

Since $\nu\left(\chi_{\alpha}\right)=\nu(\chi)=\alpha$ and $\operatorname{deg}\left(\chi_{\alpha}-\chi\right)<m$, we have:

$$
\mu\left(\chi_{\alpha}-\chi\right)=\nu\left(\chi_{\alpha}-\chi\right) \geq \alpha .
$$

By well-known criteria [7, Lem. 2.8], the two augmentations coincide: $\nu_{\chi_{\alpha}}=\nu_{\chi}$.

Therefore conditions (K3) and (K4) say that the monic polynomial $Q$ is unstable:

$$
\rho_{\alpha}(Q)=\nu_{\chi}(Q)<\nu(Q)
$$

and has minimal degree with this property. Hence, $Q$ is a MLV key polynomial for this continuous family of augmentations.

Finally, this continuous family is essential because $m_{\infty}=\operatorname{deg}(Q)>m$. Indeed, we have $\mu(Q) \leq \rho_{\alpha}(Q)<\nu(Q)$ for all $\alpha$; hence, $\operatorname{deg}(Q)=m$ would imply that $Q$ belongs to $\Phi_{\mu, \nu}$. Since obviously $\nu_{Q}(Q)=\nu(Q)$, this contradicts $(\mathrm{K} 3)$. 


\section{INVARIANTS OF LIMIT KEY POLYNOMIALS}

4.1. Basic invariants of continuous families. We keep the notation of section 3.1. Let us fix an essential continuous family $\left(\rho_{i}\right)_{i \in A}$ of a valuation $\mu$ on $K[x]$. Recall that

$$
\rho_{i}=\left[\mu ; \chi_{i}, \gamma_{i}\right], \quad \chi_{i} \in \operatorname{KP}(\mu), \gamma_{i} \in \Gamma_{\infty} \quad \text { for all } i \in A .
$$

Our aim in this section is to study certain invariants of the MLV limit key polynomials of $\left(\rho_{i}\right)_{i \in A}$, introduced in [13, Sec. 3] and [2, Sec. 4].

Let $\phi \in \mathrm{KP}_{\infty}$, and let $n=\left\lfloor m_{\infty} / m\right\rfloor$. Denote the canonical $\chi_{i}$-expansion of $\phi$ by

$$
\phi=a_{n, i} \chi_{i}^{n}+a_{n-1, i} \chi_{i}^{n-1}+\cdots+a_{1, i} \chi_{i}+a_{0, i}, \quad \forall i \in A .
$$

Since $\operatorname{deg}\left(a_{s, i}\right)<m$ for all $s, i$, all these coefficients $a_{s, i}$ are stable.

The index $t_{i}(\phi)=\max \left(S_{\rho_{i}, \chi_{i}}(\phi)\right)$ is always positive and decreases as $i$ grows. Thus, it stabilizes for $i$ sufficiently large. The stable value is known as the numerical character of $\phi$. We denote it by ${ }^{1}$

$$
t_{\infty}=t_{\infty}(\phi)
$$

This integer is a power of the characteristic exponent $p$ of the valued field $(K, v)[2$, Sec. 7].

Let $i_{0} \in A$ be an index which stabilizes $t_{\infty}$. Let us denote $t=t_{\infty}$ for simplicity. It is easy to check that the image of the coefficient $a_{t, i}$ in the graded algebra stabilizes too:

$$
a_{t, i} \sim_{\rho_{k}} a_{t, j}, \quad \text { for all } i_{0} \leq i<j \leq k .
$$

In particular, it determines a stable value

$$
\alpha_{\infty}=\alpha_{\infty}(\phi)=\rho_{i}\left(a_{t, i}\right)=\rho_{\infty}\left(a_{t, i}\right) \in \Gamma_{\infty}, \quad \forall i \geq i_{0} .
$$

Since $\rho_{i}(\phi)=\rho_{i}\left(a_{t, i} \chi_{i}^{t}\right)$, we have

$$
\rho_{i}(\phi)=\alpha_{\infty}+t_{\infty} \gamma_{i}, \quad \forall i \geq i_{0} .
$$

Proposition 4.1. Take $\phi \in \mathrm{KP}_{\infty}$ and let $i_{0} \in A$ be an index that stabilizes $t=t_{\infty}$. Then,

$$
\phi \sim_{\rho_{i}} a_{t, j} \chi_{j}^{t}, \quad \forall i_{0}<i<j .
$$

Proof. As we saw in section 3.1, $\Phi_{\rho_{k}, \rho_{\ell}}=\left[\chi_{\ell}\right]_{\rho_{k}}$ for all $k<\ell$ in $A$. Hence $\chi_{\ell}$ is a MLV key polynomial for $\rho_{k}$, for all $k \leq \ell$. Thus, it is $\rho_{k}$-minimal and Lemma 1.2 shows that

$$
\rho_{k}(\phi)=\min \left\{\rho_{k}\left(a_{s, \ell} \chi_{\ell}^{s}\right) \mid 0 \leq s\right\} \quad \text { for all } k \leq \ell \text {. }
$$

Now, denote $\alpha=\alpha_{\infty}$ and take any pair of indices $j>i>i_{0}$. Let us apply (10) for $k=i$, $\ell=j$. For $s=t$ we get the minimal value

$$
\rho_{i}\left(a_{t, j} \chi_{j}^{t}\right)=\alpha+t \gamma_{i}=\rho_{i}(\phi) .
$$

The proposition follows if we show that $\rho_{i}\left(a_{s, j} \chi_{j}^{s}\right)>\rho_{i}(\phi)$ for all the indices $s \neq t$.

For $s<t$, we apply (10) for $k=j=\ell$. We get

$$
\begin{aligned}
\rho_{\infty}\left(a_{s, j}\right)+s \gamma_{j} & =\rho_{j}\left(a_{s, j} \chi_{j}^{s}\right) \geq \rho_{j}(\phi)=\alpha+t \gamma_{j} \\
& \Longrightarrow \rho_{\infty}\left(a_{s, j}\right) \geq \alpha+(t-s) \gamma_{j}>\alpha+(t-s) \gamma_{i} .
\end{aligned}
$$

For $s>t$, we apply (10) for $k=i_{0}, \ell=j$. Since $t-s$ is a negative integer, we get

$$
\begin{aligned}
\rho_{\infty}\left(a_{s, j}\right) & +s \gamma_{i_{0}}=\rho_{i_{0}}\left(a_{s, j} \chi_{j}^{s}\right) \geq \rho_{i_{0}}(\phi)=\alpha+t \gamma_{i_{0}} \\
& \Longrightarrow \rho_{\infty}\left(a_{s, j}\right) \geq \alpha+(t-s) \gamma_{i_{0}}>\alpha+(t-s) \gamma_{i},
\end{aligned}
$$

In both cases, we deduce that

$$
\rho_{i}\left(a_{s, j} \chi_{j}^{s}\right)=\rho_{\infty}\left(a_{s, j}\right)+s \gamma_{i}>\alpha+t \gamma_{i}=\rho_{i}(\phi) .
$$

\footnotetext{
${ }^{1}$ This invariant is denoted by $t$ in [13] and by $\delta$ in [2].
} 
Residual polynomial operators of a continuous family. Let $\mu$ be a residually transcendental valuation on $K[x]$. Let $e$ be the relative ramification index of $\mu$. Take $\phi$ a MLV key polynomial for $\mu$ of minimal degree $m$. Take $a \in K[x]$ a polynomial of degree less than $m$ such that $\mu(a)=\mu\left(\phi^{e}\right)$. Let $u=\left(\operatorname{in}_{\mu} a\right)^{-1} \in \mathcal{G}_{\mu}^{*}$.

Let us recall the definition of the residual polynomial operator

$$
R=R_{\mu, \phi, u}: K[x] \longrightarrow \kappa(\mu)[y]
$$

introduced in [6, Sec. 5] (cf. Section 1.2) .

We define $R(0)=0$. For any non-zero polynomial $f \in K[x]$, consider the canonical $\phi$-expansion $f=\sum_{0 \leq s} a_{s} \phi^{s}$ and denote

$$
S(f)=S_{\mu, \phi}(f)=\left\{s \mid \mu\left(a_{s} \phi^{s}\right)=\mu(f)\right\}, \quad s_{0}=\min (S(f)) .
$$

All $s \in S(f)$ belong to a fixed class modulo $e$. Hence, $S(f) \subset\left\{s_{0}, s_{1}, \ldots, s_{d}\right\}$, where

$$
s_{j}=s_{0}+j e, 0 \leq j \leq d, \quad s_{d}=\max (S(f)) .
$$

We may write

$$
f \sim_{\mu} \sum_{s \in S(f)} a_{s} \phi^{s} \sim_{\mu} \phi^{s_{0}}\left(a_{s_{0}}+\cdots+a_{s_{j}} \phi^{j e}+\cdots+a_{s_{d}} \phi^{d e}\right),
$$

having into account only the monomials for which $s_{j} \in S(f)$. We define

$$
R(f)=\zeta_{0}+\zeta_{1} y+\cdots+\zeta_{d-1} y^{d-1}+y^{d}
$$

where the coefficients $\zeta_{j} \in \kappa(\mu)$ are defined by:

$$
\zeta_{j}= \begin{cases}\left(\operatorname{in}_{\mu} a_{s_{j}}\right)\left(\operatorname{in}_{\mu} a_{s_{d}}\right)^{-1} u^{j-d}, & \text { if } s_{j} \in S(f), \\ 0, & \text { if } s_{j} \notin S(f) .\end{cases}
$$

We are going to use this operator for the valuations $\rho_{i}$ in our essential continuous family.

Consider any $i \in A$ which is not a minimal element. For all $j>i$, write

$$
\chi_{j}=\chi_{i}+a_{j}, \quad a_{j} \in K[x], \quad \operatorname{deg}\left(a_{j}\right)<m .
$$

Since $\rho_{j}\left(\chi_{j}\right)=\gamma_{j}>\gamma_{i}=\rho_{j}\left(\chi_{i}\right)$, the stable value of $a_{j}$ is $\rho_{i}\left(a_{j}\right)=\rho_{j}\left(a_{j}\right)=\gamma_{i}=\rho_{i}\left(\chi_{i}\right)$.

We saw in section 3.1 that

$$
\left[\chi_{j}\right]_{\rho_{i}}=\Phi_{\rho_{i}, \rho_{j}}=\Phi_{\rho_{i}, \rho_{k}}=\left[\chi_{k}\right]_{\rho_{i}}, \quad \text { for all } i<j<k .
$$

Hence, $\chi_{j} \sim_{\rho_{i}} \chi_{k}$, and this implies

$$
\rho_{i}\left(a_{j}-a_{k}\right)=\rho_{i}\left(\chi_{j}-\chi_{k}\right)>\rho_{i}\left(\chi_{j}\right)=\gamma_{i} .
$$

Therefore, the following unit in the graded algebra $\mathcal{G}_{\rho_{i}}$ is well defined:

$$
u_{i}=\operatorname{in}_{\rho_{i}} a_{j}, \text { for all } j>i .
$$

The valuation $\rho_{i}$ has relative ramification index equal to one. Thus, we may consider a residual polynomial operator

$$
R_{i}=R_{\rho_{i}, \chi_{i}, u_{i}}: K[x] \longrightarrow \kappa_{i}[y],
$$

where $\kappa_{i}=\kappa\left(\rho_{i}\right)$ is the maximal subfield of $\Delta_{\rho_{i}}$.

For this choice of the pair $\chi_{i}, u_{i}$, we have by definition:

$$
R_{i}(a)=1, \quad R_{i}\left(\chi_{i}\right)=1, \quad R_{i}\left(\chi_{j}\right)=y+1, \quad \text { for all } i<j,
$$

for all $a \in K[x]$ with $\operatorname{deg}(a)<m$.

\footnotetext{
${ }^{2}$ Actually, the operator $R_{\mu, \phi, u}$ we describe here is the operator $R_{\mu, \phi, u^{-1}}$ of $[6$, Sec. 5].
} 
Since the residual operator is multiplicative [6, Cor. 5.4], we deduce immediately from Proposition 4.1 and $[6$, Cor. 5.5] that

$$
R_{i}(\phi)=R_{i}\left(a_{t, j}\right) R_{i}\left(\chi_{j}\right)^{t}=(y+1)^{t} .
$$

This result may be deduced from [2, Prop. 4.2] too.

Corollary 4.2. Take $\phi \in \mathrm{KP}_{\infty}$ and let $i_{0}$ be an index that stabilizes $t_{\infty}$. Then,

$$
S_{\rho_{i}, \chi_{i}}(\phi)=\left\{0, t_{\infty}\right\}, \quad \forall i \geq i_{0} .
$$

Proof. By the very definition of $R_{i}$, the coefficient of degree $s$ of $R_{i}(\phi)$ is zero if and only if $s \notin S_{\rho_{i}, \chi_{i}}(\phi)$. Thus, the computation of (12) shows that $0, t_{\infty}$ belong to $S_{\rho_{i}, \chi_{i}}$.

If $\operatorname{char}(k)=0$, then $t_{\infty}=1$ and the statement is obvious. If $\operatorname{char}(k)=p>0$, then $t_{\infty}=p^{e}$ for some $e \geq 0$, so that $R_{i}(\phi)=y^{t_{\infty}}+1$, and the statement follows too.

Intrinsic invariants of a continuous family. We are ready to show that the invariants $t_{\infty}, \alpha_{\infty}$ are independent of the choice of the MLV limit key polynomial $\phi$.

Lemma 4.3. For any two $\phi, \varphi \in \mathrm{KP}_{\infty}$ there exists an index $i_{0} \in A$ such that

$$
\phi \sim_{\rho_{i}} \varphi, \quad \forall i \geq i_{0} .
$$

Proof. Write $\phi=\varphi+a$ with $a \in K[x]$ of degree less than $m_{\infty}$. Since $a$ is stable, there exists an index $i_{0}$ such that $\rho_{i}(a)=\rho_{\infty}(a)$ for all $i \geq i_{0}$. We want to show that

$$
\rho_{i}(a)>\rho_{i}(\phi) \quad \forall i \geq i_{0} .
$$

In fact, $\rho_{i}(a) \leq \rho_{i}(\phi)$ leads to a contradiction:

$$
\rho_{j}(a)=\rho_{i}(a) \leq \rho_{i}(\phi)<\rho_{j}(\phi), \quad \forall j>i
$$

which implies that $\varphi$ would be stable: $\rho_{j}(\varphi)=\rho_{j}(a)=\rho_{\infty}(a)$ for all $j>i$.

The next result follows immediately from Proposition 4.1 and Lemma 4.3.

Corollary 4.4. For all $\phi, \varphi \in \mathrm{KP}_{\infty}$ we have $t_{\infty}(\phi)=t_{\infty}(\varphi)$ and $\alpha_{\infty}(\phi)=\alpha_{\infty}(\varphi)$.

Let us recall another intrinsic invariant $b_{\infty}$ of the continuous family.

Take any valuation $\nu$ on $K[x]$ such that $\nu>\rho_{i}$ for all $i$. For instance, any limit augmentation of $\left(\rho_{i}\right)_{i \in A}$.

In $[2$, Sec. 7$]$ it is shown that for a sufficiently large index $j_{0}$ one has:

$$
I\left(\chi_{j}\right)=\left\{b_{\infty}\right\}, \quad \forall j \geq j_{0},
$$

for a certain positive integer $b_{\infty}$.

Since all the polynomials $\chi_{j}$ and all their derivatives $\partial_{b}\left(\chi_{j}\right)$ are stable, it is clear that $b_{\infty}$ does not depend on the choice of the valuation $\nu$.

By Proposition 2.6, $b_{\infty}$ is a power of the characteristic exponent $p$ of $(K, v)$.

On the other hand, all the $\chi_{j}$ are key polynomials for $\nu$ such that $\nu_{\chi_{j}}=\rho_{j}$ by Theorem 2.17. Let us denote $\epsilon_{j}=\epsilon\left(\chi_{j}\right) \in\left(\Gamma_{\infty}\right)_{\mathbb{Q}}$. In [2, Cor. 7.3] it is proved that

$$
\rho_{\infty}\left(\partial_{b_{\infty}}\left(a_{j}\right)\right)>\rho_{\infty}\left(\partial_{b_{\infty}}\left(\chi_{j}\right)\right)=\rho_{\infty}\left(\partial_{b_{\infty}}\left(\chi_{k}\right)\right) \text { for all } j_{0} \leq j<k .
$$

In particular, we may consider another invariant of the essential continuous family $\left(\rho_{i}\right)_{i \in A}$, independent of $j$ and the choice of $\nu$ :

$$
\delta_{\infty}:=\rho_{\infty}\left(\partial_{b_{\infty}}\left(\chi_{j}\right)\right) \in \Gamma_{\infty}, \quad j \geq j_{0} .
$$

As a consequence we get an explicit formula for the variation of $\epsilon_{j}$ :

$$
\gamma_{j}-b_{\infty} \epsilon_{j}=\delta_{\infty}=\gamma_{k}-b_{\infty} \epsilon_{k} \Longrightarrow \epsilon_{k}-\epsilon_{j}=\frac{1}{b_{\infty}}\left(\gamma_{k}-\gamma_{j}\right),
$$

for all $k>j$. Finally, let us state and prove a basic relationship between these invariants. 
Lemma 4.5. For any $\phi \in \mathrm{KP}_{\infty}$, we have $t_{\infty} b_{\infty} \geq \operatorname{mult}(\phi)$.

Proof. Let $j$ be a sufficiently large index so that it stabilizes both $t_{\infty}$ and $b_{\infty}$. Recall that $\chi_{j}$ is a key polynomial for $\nu$ such that $\nu_{\chi_{j}}=\rho_{j}$. By Corollary $4.2, S_{\rho_{j}, \chi_{j}}(\phi)=\left\{0, t_{\infty}\right\}$. Let $b=t_{\infty} b_{\infty}$. By [2, Prop. 6.1] or [1, Prop. 14],

$$
\rho_{j}\left(\partial_{b}(\phi)\right)=\rho_{j}(\phi)-b \epsilon_{j} .
$$

In particular, $\partial_{b}(\phi) \neq 0$, so that $b \geq \operatorname{mult}(\phi)$.

4.2. Vertically bounded continuous families. Let us recall Hahn's embedding theorem for ordered groups. A basic reference for this result is [11].

Let $\Lambda$ be an abelian (totally) ordered group. A subgroup $H \subset \Lambda$ is convex if it satisfies

$$
0<\beta<\gamma, \quad \gamma \in H \Longrightarrow \beta \in H,
$$

for all $\beta, \gamma \in \Lambda_{>0}$.

For any $\gamma \in \Lambda$ we denote by $H_{\gamma}$ the convex subgroup generated by $\gamma$. That is, $H_{\gamma}$ is the intersection of all convex subgroups of $\Lambda$ that contain $\gamma$. The convex subgroups of the form $H_{\gamma}$ are said to be principal.

The principal convex subgoups of $\Lambda$ are totally ordered by inclusion. Let us denote by

$$
I=\operatorname{Prin}(\Lambda)
$$

the set of non-zero convex principal subgroups of $\Lambda$, ordered by decreasing inclusion.

Formally, we consider $I$ as an abstract totally ordered set parameterizing the non-zero principal convex subgoups. For any $i \in I$ we denote by $H_{i}$ the corresponding principal convex subgroup. Note that

$$
i \leq j \Longleftrightarrow H_{i} \supset H_{j}
$$

Denote by $\mathbb{R}_{\text {lex }}^{I}$ the Hahn product; that is, $\mathbb{R}_{\text {lex }}^{I} \subset \mathbb{R}^{I}$ is the subgroup of the cartesian product $\mathbb{R}^{I}$ formed by the elements $\gamma=\left(x_{i}\right)_{i \in I}$ whose support

$$
\operatorname{supp}(\gamma)=\left\{i \in I \mid x_{i} \neq 0\right\} \subset I
$$

is a well-ordered subset, with respect to the ordering induced by $I$. It makes sense to consider the lexicographical ordering on $\mathbb{R}_{\text {lex }}^{I}$.

By Hahn's theorem, there is an embedding of ordered groups

$$
\Lambda \hookrightarrow \Lambda_{\mathbb{Q}} \longleftrightarrow \mathbb{R}_{\text {lex }}^{I},
$$

such that the natural mapping

$$
\operatorname{Prin}(\Lambda) \longrightarrow \operatorname{Prin}\left(\mathbb{R}_{\text {lex }}^{I}\right), \quad H_{\gamma} \mapsto\left(H_{\gamma}\right)_{\mathbb{R}}=\text { convex subgroup of } \mathbb{R}_{\text {lex }}^{I} \text { generated by } \gamma
$$

is an isomorphism of ordered sets.

Definition 4.6. Consider a subset $S \subset \Lambda_{>0}$ of positive elements in $\Lambda$, and let $H_{S}$ be the convex subgroup of $\Lambda$ generated by $S$.

We say that $S$ is vertically bounded (VB) if $S$ admits an upper bound in $H_{S}$.

We say that $S$ is horizontally bounded $(H B)$ if $S$ has no upper bounds in $H_{S}$, but it admits an upper bound in $\Lambda$.

We say that $S$ is unbounded (UB) if $S$ admits no upper bounds in $\Lambda$.

Clearly, any such set $S$ falls in one, and only one, of the three cases VB, HB or UB.

Horizontally bounded sets occur only in ordered groups of rank greater than one.

The next table displays some examples in the ordered group $\Lambda=\mathbb{Q}_{\text {lex }}^{2}$. In this case, all convex subroups are principal and $I=\{1,2\}$. The non-zero convex subgroups are $H_{1}=\Lambda$, $H_{2}=\{0\} \times \mathbb{Q}$. The set $S$ is a sequence $S=\left(\gamma_{n}\right)_{n \in \mathbb{N}}$. 


\begin{tabular}{|c|c|c|}
\hline$\gamma_{n}$ & $H_{S}$ & boundedness \\
\hline$(0,1-(1 / n))$ & $\{0\} \times \mathbb{Q}$ & VB \\
\hline$(1, n)$ & $\Lambda$ & VB \\
\hline$(0, n)$ & $\{0\} \times \mathbb{Q}$ & HB \\
\hline$(n, 0)$ & $\Lambda$ & UB \\
\hline
\end{tabular}

Lemma 4.7. Let $S=\left\{\gamma_{\alpha} \mid \alpha \in A\right\}$ be a subset of positive elements in $\Lambda$. Let $H_{S}$ be the convex subgroup of $\Lambda$ generated by $S$. Then, the following conditions are equivalent.

(1) $S$ is vertically bounded.

(2) For all $q \in \mathbb{Q}, q>1$, there exists $\alpha \in A$ such that $q \gamma_{\alpha}>S$ in $\Lambda_{\mathbb{Q}}$.

In this case, $H_{S}$ is a principal convex subgroup.

Proof. Let us see that (2) implies (1). From $q \gamma_{\alpha}>S$ we deduce that $n \gamma_{\alpha}>S$ for any integer $n \geq q$. Thus, $S$ admits an upper bound $n \gamma_{\alpha} \in H_{S}$.

Let us show that (1) implies (2). If $\gamma \in H_{S}$ satisfies $\gamma>S$, then $S \subset H_{\gamma}$ by the convexity of $H_{\gamma}$. Hence, $H_{S}=H_{\gamma}$ is a principal convex subgroup.

Let $i \in I$ such that $H_{i}=H_{S}$. Then,

$$
\left(H_{S}\right)_{\mathbb{R}}=\left\{\left(x_{j}\right) \in \mathbb{R}_{\text {lex }}^{I} \mid x_{j}=0, \forall j<i\right\} .
$$

Thus, we may write

$$
\gamma=(0 \cdots 0 x \star \star \cdots), \quad \gamma_{\alpha}=\left(0 \cdots 0 x_{\alpha} \star \star \cdots\right), \quad \forall \alpha \in A,
$$

where $x, x_{\alpha} \in \mathbb{R}$ are the $i$-th coordinates. They satisfy $0 \leq x_{\alpha} \leq x$ for all $\alpha \in A$, and $x_{\alpha}>0$ for some $\alpha$ (otherwise $S$ would not generate $H_{i}$ ).

Consider $b=\sup \left\{x_{\alpha} \mid \alpha \in A\right\}$. For any given $q \in \mathbb{Q}, q>1$, there exists $\alpha \in A$ such that $b<q x_{\alpha}$. Hence, $q \gamma_{\alpha}>S$.

Definition 4.8. Let $\left(\rho_{i}\right)_{i \in A}$ be an essential continuous family of augmentations such that $A$ is well-ordered. Let $i_{0}$ be the first element in $A$ that stabilizes $t_{\infty}$, and consider the set

$$
S=\left\{\gamma_{i}-\gamma_{i_{0}} \mid i>i_{0}\right\} .
$$

We say that $\left(\rho_{i}\right)_{i \in A}$ is vertically bounded, horizontally bounded or unbounded according to the boundedness status of $S$ introduced in Definition 4.6.

Theorem 4.9. Let $\left(\rho_{i}\right)_{i \in A}$ be a vertically bounded essential continuous family of augmentations. Then, $m_{\infty}=m t_{\infty}$ and $\alpha_{\infty}=0$.

Proof. Denote $t=t_{\infty}, \alpha=\alpha_{\infty}$, and let $i_{0}$ be the first index that stabilizes $t_{\infty}$.

The set $S=\left(\gamma_{i}-\gamma_{i_{0}}\right)_{i>i_{0}}$ admits an upper bound in the principal convex subgroup generated by $S$. By Lemma 4.7, there exists an index $j>i_{0}$ such that

$$
\frac{t+1}{t}\left(\gamma_{j}-\gamma_{i_{0}}\right)>\left(\gamma_{i}-\gamma_{i_{0}}\right), \quad \forall i>i_{0} .
$$

Take any $\phi \in \mathrm{KP}_{\infty}$, and let $n=\left\lfloor m_{\infty} / m\right\rfloor$. Consider the canonical $\chi_{j}$-expansion of $\phi$,

$$
\phi=a_{n, j} \chi_{i}^{n}+a_{n-1, j} \chi_{i}^{n-1}+\cdots+a_{1, j} \chi_{i}+a_{0, j} .
$$

Claim. $\phi \sim_{\rho_{i}} a_{t, j} \chi_{j}^{t}+\cdots+a_{1, j} \chi_{j}+a_{0, j}, \quad \forall i>i_{0}$.

To prove the Claim we must show that

$$
\rho_{i}\left(a_{s, j} \chi_{j}^{s}\right)>\rho_{i}(\phi)=\alpha+t \gamma_{i}, \quad \forall s>t, \quad \forall i>i_{0} .
$$

This holds whenever $i<j$ by Proposition 4.1. Thus, we may assume that $i \geq j$. In this case, $\rho_{i}\left(\chi_{j}\right)=\gamma_{j}$. 
Take any $s>t$. We saw in (11) that $\rho_{\infty}\left(a_{s, j}\right) \geq \alpha+(t-s) \gamma_{i_{0}}$. Hence,

$$
\rho_{i}\left(a_{s, j} \chi_{j}^{s}\right)=\rho_{\infty}\left(a_{s, j}\right)+s \gamma_{j} \geq \alpha+(t-s) \gamma_{i_{0}}+s \gamma_{j}=\alpha+t \gamma_{i_{0}}+s\left(\gamma_{j}-\gamma_{i_{0}}\right) .
$$

We want to show that $\alpha+t \gamma_{i_{0}}+s\left(\gamma_{j}-\gamma_{i_{0}}\right)>\alpha+t \gamma_{i}$, which amounts to

$$
s\left(\gamma_{j}-\gamma_{i_{0}}\right)>t\left(\gamma_{i}-\gamma_{i_{0}}\right),
$$

and this follows from (14). This ends the proof of the Claim.

By the Claim, the polynomial $F=a_{t, j} \chi_{j}^{t}+\cdots+a_{1, j} \chi_{j}+a_{0, j}$ is unstable. By the minimality of $m_{\infty}=\operatorname{deg}(\phi)$, we must have $F=\phi$.

Since the coefficients $a_{s, j}$ have degree less than $m=\operatorname{deg}\left(\rho_{i_{0}}\right)$, those which are non-zero determine units in the graded algebra $\mathcal{G}_{\rho_{i_{0}}}$. Conversely, any unit in $\mathcal{G}_{\rho_{i_{0}}}$ is the initial term of a polynomial of degree less than $m$ [6, Prop. 3.5]. Therefore, there exist polynomials $b, c_{0}, \ldots, c_{t-1} \in K[x]$, all of degree less than $m$, such that:

$$
b a_{t, j} \sim_{\rho_{i_{0}}} 1, \quad b a_{s, j} \sim_{\rho_{i_{0}}} c_{s}, \quad 0 \leq s<t .
$$

Since $\rho_{i_{0}}\left(c_{s}\right)=\rho_{\infty}\left(c_{s}\right)$ for all $s$, we have

$$
b a_{t, j} \sim_{\rho_{i}} 1, \quad b a_{s, j} \sim_{\rho_{i}} c_{s}, \quad 0 \leq s<t,
$$

for all $i>i_{0}$. By the Claim, we deduce that

$$
b \phi \sim_{\rho_{i}} \chi_{j}^{t}+c_{t-1} \chi_{j}^{t-1}+\cdots+c_{0}, \quad \forall i>i_{0} .
$$

Since $b \phi$ is clearly unstable, this implies that the polynomial of degree $m t$,

$$
\chi_{j}^{t}+c_{t-1} \chi_{j}^{t-1}+\cdots+c_{0},
$$

is unstable too. By the minimality of $m_{\infty}=\operatorname{deg}(\phi)=\operatorname{deg}\left(a_{t, j}\right)+m t$, we deduce that $\operatorname{deg}\left(a_{t, j}\right)=0$, which implies $a_{t, j}=1$ because $\phi$ is monic.

This proves that $m_{\infty}=m t$ and $\alpha=\rho_{\infty}\left(a_{t, j}\right)=0$.

Corollary 4.10. For all VB essential continuous families, we have $t_{\infty}>1$.

Therefore, there are no VB essential continuous families at all, if $\operatorname{char}(k)=0$.

Theorem 4.9 was proved for $\rho$ of finite rank in [13, Sec. 3], and for $\rho$ of rank one in [2, Sec. 5]. Actually, both proofs are valid for arbitrary rank, once the right definition of vertically bounded chain is introduced. We followed the approach of Vaquié in [13].

\subsection{Invariants of unbounded continuous families.}

Theorem 4.11. Let $\left(\rho_{i}\right)_{i \in A}$ be an essential continuous family. If the set $\left(\gamma_{i}\right)_{i \in A}$ is unbounded in $\Gamma_{\infty}$, then

$$
t_{\infty} b_{\infty}=\operatorname{mult}(\phi), \quad \forall \phi \in \mathrm{KP}_{\infty} .
$$

Proof. Let $\nu$ be any valuation on $K[x]$ such that $\nu>\rho_{i}$ for all $i$. Recall that all the $\chi_{i}$ are key polynomials for $\nu$ such that $\nu_{\chi_{i}}=\rho_{i}$. Denote $\epsilon_{i}=\epsilon\left(\chi_{i}\right)$ for all $i$.

Let $\phi \in \mathrm{KP}_{\infty}\left(\left(\rho_{i}\right)_{i \in A}\right)$. Denote

$$
b=\operatorname{mult}(\phi), \quad t=t_{\infty}, \quad \alpha=\alpha_{\infty} .
$$

Since $\partial_{b}(\phi)$ has degree less than $m_{\infty}$, it is a stable polynomial. Let $i_{0} \in A$ be any index that stabilizes $t_{\infty}, b_{\infty}$ and $\rho_{\infty}\left(\partial_{b}(\phi)\right)$. By definition,

$$
\epsilon_{i}=\frac{\nu\left(\chi_{i}\right)-\nu\left(\partial_{b_{\infty}}\left(\chi_{i}\right)\right)}{b_{\infty}}=\frac{\gamma_{i}-\delta_{\infty}}{b_{\infty}}, \quad \forall i \geq i_{0},
$$

where $\delta_{\infty}$ is the invariant introduced in (13). 
By (i) of Proposition 2.11, for all $i \geq i_{0}$ we have

$\rho_{i}\left(\partial_{b}(\phi)\right) \geq \rho_{i}(\phi)-b \epsilon_{i}=\alpha+t \gamma_{i}-b \epsilon_{i}=\alpha+t \gamma_{i}-\frac{b}{b_{\infty}}\left(\gamma_{i}-\delta_{\infty}\right)=\alpha+\left(t-\frac{b}{b_{\infty}}\right) \gamma_{i}+\frac{b \delta_{\infty}}{b_{\infty}}$.

From this inequality we deduce

$$
\left(t-\frac{b}{b_{\infty}}\right) \gamma_{i} \leq \rho_{\infty}\left(\partial_{b}(\phi)\right)-\alpha-\frac{b \delta_{\infty}}{b_{\infty}}, \quad \forall i \geq i_{0}
$$

Since $\partial_{b}(\phi) \neq 0$, we have necessarily $t b_{\infty} \leq b$ : otherwise, the set $\left(\gamma_{i}\right)_{i \in A}$ would admit an upper bound in $\left(\Gamma_{\infty}\right)_{\mathbb{Q}}$, and hence in $\Gamma_{\infty}$, contradicting our assumption.

This proves $t b_{\infty} \leq b$, and the equality follows from Lemma 4.5.

Corollary 4.12. If $\operatorname{char}(K)=0$ and the set $\left(\gamma_{i}\right)_{i \in A}$ is unbounded in $\Gamma_{\infty}$, then

$$
t_{\infty}=b_{\infty}=1 \text {. }
$$

Proof. If $\operatorname{char}(K)=0$, then $\operatorname{mult}(\phi)=1$.

In W. Mahboub PhD thesis [5], some examples of continuous families and limit key polynomials are exhibited. Among the HB ones, there are some examples in which the inequality $t_{\infty} b_{\infty} \geq \operatorname{mult}(\phi)$ is an equality (Examples 5.3.1, 5.3.2, and 5.3.3), and one where it is an strict inequality (Example 5.3.4).

On the other hand, any monic irreducible polynomial $\phi \in K[x]$ which determines an extension of $K$ with defect, is a limit key polynomial of a suitable continuous family. In the survey [3] of F.-V. Kulhmann, some VB examples are exhibited. Among them, we find some cases where the inequality $t_{\infty} b_{\infty} \geq \operatorname{mult}(\phi)$ is an equality (Example 3.14), and some where it is an strict inequality (Examples 3.12, 3.17, 3.20 and 3.22).

Acknowledgements. We thank the anonymous referee for her/his extremely accurate report, which led to an improvement in the presentation of the results.

\section{REFERENCES}

[1] J. Decaup, W. Mahboub, M. Spivakovsky, Abstract key polynomials and comparison theorems with the key polynomials of MacLane-Vaquié, Illinois J. Math. 62, Number 1-4 (2018), 253-270.

[2] F.J. Herrera Govantes, W. Mahboub, M.A. Olalla Acosta, M. Spivakovsky, Key polynomials for simple extensions of valued fields, preprint, arXiv:1406.0657v4 [math.AG], 2018.

[3] F.-V. Kuhlmann, The defect, in: Commutative Algebra - Noetherian and non-Noetherian perspectives, Marco Fontana, Salah-Eddine Kabbaj, Bruce Olberding and Irena Swanson (eds.), Springer 2011.

[4] S. MacLane, A construction for absolute values in polynomial rings, Trans. Amer. Math. Soc. 40 (1936), pp. 363-395.

[5] W. Mahboub, Une construction explicite de polynômes-clé pour des valuations de rang fini, Thèse de Doctorat, Institut de Mathématiques de Toulouse, Novembre 2013.

[6] E. Nart, Key polynomials over valued fields, Publ. Mat. 64 (2020), 3-42.

[7] E. Nart, MacLane-Vaquié chains of valuations on a polynomial ring, arXiv:1911.01714v3 [math.AG].

[8] J. Novacoski, M. Spivakovsky, On the local uniformization problem, Banach Center Publ. 108 (2016), 231-238.

[9] J. Novacoski, M. Spivakovsky, Key polynomials and pseudo-convergent sequences, J. Algebra 495 (2018), 199-219.

[10] J. Novacoski, M. Spivakovsky, Key polynomials and minimal pairs, J. Algebra 523 (2019), 1-14.

[11] P. Ribenboim, Théorie des valuations, Presses Univ. Montréal, Montréal, 1968.

[12] J.-C. San Saturnino, Defect of an extension, key polynomials and local uniformization, J. Algebra 481 (2017), 91-119.

[13] M. Vaquié, Famille admisse associée à une valuation de $K[x]$, Singularités Franco-Japonaises, Séminaires et Congrés 10, SMF, Paris (2005), Actes du colloque franco-japonais, juillet 2002, édité par Jean-Paul Brasselet et Tatsuo Suwa, 391-428.

[14] M. Vaquié, Extension d'une valuation, Trans. Amer. Math. Soc. 359 (2007), no. 7, 3439-3481. 
Institut de Robòtica i Informàtica Industrial (IRI, CSIC-UPC) And Departament de Matemàtiques, Universitat Politècnica de Catalunya · Barcelonatech, Av. Diagonal, 647, 08028 BARCElona, Spain

Email address: Maria.Alberich@upc.edu

Ben Gurion Univ Negev, Dept Math, POB 653, IL-84105 Beer Sheva, Israel

Email address: albertof .boix@gmail.com

Departament de Matemàtica Aplicada IV, Escola Politècnica Superior d’Enginyeria de Vilanova i la Geltrú, Av. Víctor Balaguer s/n. E-08800 Vilanova i la Geltrú, Catalonia

Email address: julio.fernandez.g@upc.edu, jordi.guardia-rubies@upc.edu

Departament de Matemàtiques, Universitat Autònoma de Barcelona, Edifici C, E-08193 Bellaterra, Barcelona, Catalonia

Email address: nart@mat.uab.cat, jroe@mat.uab.cat 